\title{
Brain Connectivity Mapping using Riemannian Geometry, Control Theory and PDEs
}

\author{
Christophe Lenglet * Emmanuel Prados ${ }^{\dagger} \quad$ Jean-Philippe Pons ${ }^{\ddagger}$ \\ Rachid Deriche ${ }^{\S} \quad$ Olivier Faugeras $₫$
}

July 7, 2008

Abstract

We introduce an original approach for the cerebral white matter connectivity mapping from Diffusion Tensor Imaging (DTI). Our method relies on a global modeling of the acquired Magnetic Resonance Imaging (MRI) volume as a Riemannian manifold whose metric directly derives from the diffusion tensor. These tensors will be used to measure physical three-dimensional distances between different locations of a brain diffusion tensor image. The key concept is the notion of geodesic distance that will allow us to find optimal paths in the white matter. We claim that such optimal paths are reasonable approximations of neural fiber bundles. The geodesic distance function can be seen as the solution of two theoretically equivalent but, in practice, significantly different problems in the Partial Differential Equation framework: An initial value problem which is intrinsically dynamic, and a boundary value problem which is, on the contrary, intrinsically stationary. The two approaches have very different properties which make them more or less adequate for our problem and more or less computationally efficient. The dynamic formulation is quite easy to implement but has several practical drawbacks. On the contrary, the stationary formulation is much more tedious to implement, however we will show that it has many virtue which make it more suitable for our connectivity mapping problem. Finally, we will present different possible measures of connectivity, reflecting the degree of connectivity between different regions of the brain. We will illustrate these notions on synthetic and real DTI datasets.

\footnotetext{
*Siemens Corporate Research, Princeton, NJ, USA (Christophe.Lenglet@siemens.com)
}

The first author was affiliated with INRIA Sophia Antipolis when the work described in this manuscript was done.

${ }^{\dagger}$ Perception Team, INRIA Rhône-Alpes, France (Emmanuel.Prados@inrialpes.fr)

${ }_{\ddagger}$ CERTIS Lab., École Nationale des Ponts et Chaussées, France (Jean-Philippe.Pons@certis.enpc.fr)

§Odyssée Lab., INRIA Sophia Antipolis, France (Rachid.Deriche@sophia.inria.fr)

ฯ Odyssée Lab., INRIA Sophia Antipolis, France (Olivier.Faugeras@sophia.inria.fr) 
Keywords: Brownian motion, diffusion process, control theory, Partial Differential Equations, Riemannian manifolds, Hamilton-Jacobi-Bellman equations, level set, Fast Marching methods, anisotropic Eikonal equation, intrinsic distance function, brain connectivity mapping, Diffusion Tensor Imaging

\section{Introduction}

Diffusion MRI is an exciting extension of MRI introduced in the mid-1980s by Bihan and Breton (1985), Merboldt et al. (1985) and Taylor and Bushell (1985). It provides a unique and sensitive probe for the architecture of biological tissues. The key idea behind diffusion MRI is that water diffusion in structured tissues, like the brain white matter, reflects their architecture at a microscopic scale. This is due to the fact that molecular motion is favored in directions aligned with fiber bundles and hindered in the orthogonal orientation. Measuring, at each voxel, the effect of that very motion on the MR signal for a number of directions provides an exquisite insight into the local orientation of fibers. Shortly after the first acquisitions of images characterizing the anisotropic diffusion of water molecules in vivo by Moseley et al. (1990) and Osment et al. (1990), the Diffusion Tensor (DT) formalism was introduced by Basser et al. (1994). Diffusion Tensor Imaging (DTI) features an analytic means to precisely describe the three-dimensional nature of anisotropy in tissues. The diffusion tensor model encapsulates the averaged diffusion properties of water molecules (inside a typical 1-3mm sized voxel) as the $3 \times 3$ covariance matrix of a Gaussian distribution. DTI has been extensively used to study a wide range of neurological disorders like cerebrovascular diseases, multiple sclerosis, Alzheimer's or Parkinson's disease, schizophrenia, brain tumors...etc. It has also been very useful to investigate brain development or the effect of aging, the structure of cerebral anatomo-functional networks, the thalamus or striatum and of various fiber bundles. Tractography and connectivity mapping techniques are at the core of all those studies.

Following Mori et al. (1999), various local approaches, often named deterministic trac- 
tography, have been proposed to tackle this problem. They are based on line propagation techniques and rely on the fact that the eigenvector of the diffusion tensor associated with the major eigenvalue, provides a relatively accurate estimate of the fibers' orientation at each voxel. These methods (see for instance Jones et al. (1999), Basser et al. (2000), Vemuri et al. (2001) , Mori and van Zijl (2003), Lazar et al. (2003)) may be refined to incorporate some natural constraints such as regularity or local uncertainty and avoid being stopped in regions of low anisotropy. All these efforts aim to overcome the intrinsic ambiguity of diffusion tensor data arising from partial volume effects at locations of fiber crossings (Alexander et al. (2001)). They provide relatively accurate models of the white matter macroscopic bundles.

More recent work can be divided into approaches based on Bayesian models, diffusion simulation and geometric methods, the latter being essentially based on front-propagation techniques. These methods are both more robust to noise and partial volume effects than deterministic tractography, and naturally yield probability/scalar measures which can be used to evaluate the degree of connectivity between voxels.

In Behrens et al. (2003), Parker et al. (2003), Parker and Alexander (2003), Bjornemo et al. (2002), Friman and Westin (2005), Friman et al. (2006) stochastic tractography algorithms were introduced by modeling the uncertainty of the local fiber orientation. Through uncertainty propagation, they can also provide a powerful means to evaluate the probability of connection between points of the white matter. However, the intrinsic drawback of these methods is their computational complexity since it is necessary to resort to Markov Chain Monte Carlo methods or, as in Friman et al. (2006), to evaluate probability density functions at enough locations of the space of interest. More recently, Zhang et al. (2007) proposed to use sequential Monte Carlo techniques and von Mises-Fisher distributions to efficiently model and sample from the noisy directional data, as in McGraw and Nadar (2007). Additionally, McGraw and Nadar (2007) proposed to exploit the highly parallel nature of Graphics Processing Units (GPU) to simultaneously generate several (independent) potential fiber pathways. Other probabilistic approaches like Koch et al. (2001), Lazar and Alexander 
(2002), Mangin et al. (2002), Hagmann et al. (2003), Lazar and Alexander (2005) use techniques such as perturbation methods or random walk to assess uncertainty in tractography. Diffusion simulation approaches like Batchelor et al. (2001), O’Donnell et al. (2002), Chung et al. (2003), Kang et al. (2005), Yörük et al. (2005) or Hageman et al. (2006) use the full diffusion tensor to simulate a diffusion process or a fluid flow. The resulting concentration or flow maps can be used to evaluate some degree of connectivity between regions of interest. Finally, geometric methods use either the level set (see Osher and Sethian (1988)) framework in O’Donnell et al. (2002), or in our preliminary work Lenglet et al. (2004), Fast Marching Method (see Tsitsiklis (1995), Sethian (1996b)) in Parker et al. (2002b;a), in our other preliminary work Prados et al. (2006a;b) and recently in a similar study Jbabdi et al. (2008) , Fast Sweeping Method in Jackowski et al. (2005) or Fast Iterative Method in Jeong and Whitaker (2007), Fletcher et al. (2007) to evolve a front by using a Riemannian metric derived from the diffusion tensor. In Jbabdi et al. (2008), the authors extended a variant of Tsitsiklis (1995), presented in Qingfen (2003), by deriving an analytic solution to the optimization problem that needs to be solved for updating the distance function. In Pichon et al. (2003), the authors proposed to use curve evolution techniques (through multiple level set functions) to recover fibers. As introduced by Tournier et al. (2003) and Campbell et al. (2005), it is also possible to adapt the level set based front propagation techniques to take advantage of the information provided by high angular resolution diffusion MRI (HARDI) (Tuch (2002)). Using a Fast Sweeping Method, Pichon et al. (2005) and Melonakos et al. (2007) generalized the front propagation techniques to HARDI by using a Finsler (rather than a Riemannian) metric in the Hamilton-Jacobi-Bellman problem to define and compute optimal paths in the white matter.

As we will see in the following, level set approaches to connectivity mapping tend to be somewhat inefficient since, even with a narrow-band implementation, the number of points where the evolution speed has to be evaluated greatly increases as the surface grows. We will show that this class of methods is also prone to interpolation errors at the boundary of the domain. For our brain connectivity problem, this may lead to erroneous connections in 
highly convoluted areas and will lead us to consider another approach, namely the stationary formulation of the Eikonal equation.

Contributions of this paper Our contribution is threefold:

1. First, we describe how to use the diffusion tensor as a Riemannian metric. We clarify the link between Brownian motion and diffusion MRI and expose how the knowledge of the diffusion properties of water molecules on a manifold $\mathcal{M}=\left(\mathbb{R}^{3}, g\right)$ is sufficient to infer its geometry.

2. Next, we present the dynamic formulation of the anisotropic Eikonal equation (within the level set framework), show how to solve it and to estimate neural fiber bundles by back-propagation.

3. Finally, we demonstrate that we can greatly improve the computational time and the robustness of the previous method by recasting the intrinsic geodesic distance computation into a stationary problem (with a Fast Marching implementation). In fact, it is possible to solve, quickly and simultaneously, for the geodesic distance, the optimal vector field (optimal dynamics) corresponding to the geodesics velocities and the statistics, along those curves, of a local connectivity measure. To our knowledge, the proposed GCM (for "Geodesic Connectivity Mapping”) algorithm is faster than any other existing CPU-based method. Moreover, our algorithm can naturally work within a mask of the white matter (accurately obtained by segmentation of a high-resolution anatomical MRI for instance). As we will show, this is crucial for the applications of interest since we must strictly respect the geometry of the cortical foldings or white matter / cerebrospinal fluid (CSF) interface to recover meaningful connections. For a region of interest $x_{0}$ (i.e. a point of the white matter), our GCM method generates statistics of a local connectivity measure along the geodesics linking $x_{0}$ to other locations of the brain. This can be used to discriminate likely and unlikely connections.

Organization of the paper Section 2 justifies the use of the diffusion tensor as a metric. Next, section 3 details the dynamic formulation of our geodesic distance computation problem 
and proposes a numerical scheme within the level set framework. Section 4 reformulates the anisotropic Eikonal equation as an optimal control problem which leads, in section 5, to a fast numerical algorithm for the distance function approximation. We finally introduce a general local connectivity measure whose statistics along the optimal paths may be used to evaluate the degree of connectivity of any pair of voxels. All those quantities can be computed simultaneously in a Fast Marching framework, directly yielding the connectivity maps. We illustrate our technique by showing results on real and synthetic datasets in section 6 .

\section{White Matter as a Riemannian Manifold}

Diffusion tensor, as thermal or electrical conductivity tensors, belongs to the broader class of general effective property tensors and is defined as the proportionality term between an averaged generalized intensity $B$ and an averaged generalized flux $F$. In our particular case of interest, $B$ is the gradient of the concentration $\nabla C$ and $F$ is the mass flux $J$ such that Fick's (first) law holds: $J=-\mathbf{D} \nabla C$. By considering the conservation of mass, the general diffusion equation (Fick's second law) is readily obtained:

$$
\frac{\partial C}{\partial t}=\nabla \cdot(\mathbf{D} \nabla C)
$$

In an isotropic and homogeneous medium, equation 1 boils down to the heat equation ( $\mathbf{D}$ is the identity matrix) whose solution is well-known: a Gaussian distribution. In anisotropic tissues (like the cerebral white matter), the motion of water molecules varies in direction depending on obstacles (such as axonal membranes). We recall that the symmetric and positive definite tensor D has been related in Einstein (1956) to the root mean square of the diffusion

displacement $\mathbf{R}$ during time $\tau$ by $\mathbf{D}=\frac{1}{6 \tau}\left\langle\mathbf{R} \mathbf{R}^{T}\right\rangle$ (see section 3.3.2 of Lenglet (2006)) and is thus an effective means to characterize the diffusion properties of anisotropic media.

For an unbounded anisotropic homogeneous medium, the minimal fundamental solution of equation 1 with initial concentration $\lim _{t \rightarrow 0} p\left(\mathbf{r} \mid \mathbf{r}_{0}, t\right)=\delta\left(\mathbf{r}-\mathbf{r}_{0}\right)$ is also well-known and expressed as:

$$
p\left(\mathbf{r} \mid \mathbf{r}_{0}, \tau\right)=\frac{1}{\sqrt{(4 \pi \tau)^{3}|\mathbf{D}|}} \exp \left(-\frac{\left(\mathbf{r}-\mathbf{r}_{0}\right)^{T} \mathbf{D}^{-1}\left(\mathbf{r}-\mathbf{r}_{0}\right)}{4 \tau}\right)
$$


However, when dealing with anisotropic and inhomogeneous media (like the cerebral white matter), the derivation of an explicit solution for the fundamental solution $p$ becomes difficult and is often not possible since the tensor $\mathbf{D}$ depends on the location $x \in \Omega$ and will be noted $D_{x}$ now on. It turns out that, in fact, this derivation is not necessary. It is indeed possible to reformulate equation 1 by considering the diffusion process to occur on a Riemannian manifold $\mathcal{M}$ and not a linear space as up to now. The basic idea is to rely on the metric (varying from point to point) to naturally take into account the space anisotropy and inhomogeneity (ie. the spatial variation of $D_{x}$ ). Intuitively, we transform an anisotropic and inhomogeneous diffusion on a linear space $\mathbb{R}^{n}$ into an isotropic and homogeneous diffusion on a nonlinear space $\mathcal{M}$.

The counterpart of the Laplacian on a Riemannian manifold is classically defined as the divergence of the gradient. It is known as the Laplace-Beltrami operator which, for a scalar function $f$, writes:

$$
\Delta_{\mathcal{M}} f=\operatorname{div}(\operatorname{grad} f)
$$

The intrinsic divergence and gradient operators grad and div are defined as follows in local coordinates:

$$
(\operatorname{grad} f)^{i}=g^{i j} \frac{\partial f}{\partial \mathbf{x}^{j}} \quad \forall i, j=1, \ldots, n
$$

and

$$
\operatorname{div} X=\frac{1}{\sqrt{|\operatorname{det}(G)|}} \frac{\partial}{\partial \mathbf{x}^{i}}\left(\sqrt{|\operatorname{det}(G)|} X^{i}\right)
$$

where $g^{i j}$ denotes the components of the inverse of the metric tensor $G, X$ is a vector field on $\mathcal{M}$ and Einstein notation has been used. Putting things together, the Laplace-Beltrami operator can be written in local coordinates as:

$$
\Delta_{\mathcal{M}} f=\frac{1}{\sqrt{|\operatorname{det}(G)|}} \frac{\partial}{\partial \mathbf{x}^{i}}\left(\sqrt{|\operatorname{det}(G)|} g^{i j} \frac{\partial f}{\partial \mathbf{x}^{j}}\right)=\frac{1}{\sqrt{|\operatorname{det}(G)|}} \frac{\partial}{\partial \mathbf{x}^{i}}\left(\sqrt{|\operatorname{det}(G)|} G^{-1} \nabla f\right)
$$

Comparing this expression with equation 1 , we can see that the diffusion tensor $\mathbf{D}$ plays the same role as the inverse of the Riemannian metric, $G^{-1}$. This relation is actually at the basis of many works on stochastic processes on Riemannian manifolds such as de Lara (1995) or 
Darling (1998) and has been proposed for DTI in Wedeen et al. (1995), O’Donnell et al. (2002), Fletcher et al. (2007) and Jbabdi et al. (2008). In the following, we will use the inverse of the diffusion tensor as a Riemannian metric of the cerebral white matter. As explained in previous works, the intuition behind this choice is rather simple. Diffusion is the highest along the fibers. Inverting the tensor results in the largest eigenvalue becoming the smallest and consequently, the shortest geodesic distance is parallel to fibers. Hence shortest paths should approximate white matter fiber pathways. As already pointed out by several authors such as Tournier et al. (2003), Lazar et al. (2003), Fletcher et al. (2007) and Jbabdi et al. (2008), this metric may not be suitable in all circumstances and Jbabdi et al. thoroughly investigated the practical consequences of this choice. They show that it can generate "shortcuts" if tensors are not sharp enough, precisely because of the fact that front propagation algorithms allow to deviate from the major direction of diffusion. This is one of their strengths but it also has to be carefully dealt with to prevent the estimation of artificial connections as the front may leak into unexpected regions. As described in our other work Descoteaux et al. (2007), we can avoid this kind of configuration by working with fiber tensors instead of diffusion tensors. It is essentially a deconvolution (sharpening) procedure for tensors, similar to raising tensors to a certain power (as in Tournier et al. (2003), Lazar et al. (2003), Fletcher et al. (2007) for instance) but physically well-defined and numerically more stable. In this paper, we do not investigate this important but separate issue which must be addressed with diffusion phan-

toms such as those described in Perrin et al. (2005) to assess the anatomical correctness of tractography results. As in Jbabdi et al. (2008), we concentrate on the numerical schemes for the computation of the geodesic distance and optimal paths by using the inverse of the diffusion tensor, and derive a new connectivity measure that quantifies the likelihood of each path.

\section{A Level Set Formulation for the Geodesic Distance}

We are now concerned with the computation of the distance function $u$ from a closed, nonempty subset $K$ of the 3-dimensional Riemannian manifold $\mathcal{M}=\left(\mathbb{R}^{3}, g\right)$. In the remaining, 
$K$ will be restricted to a single point $x_{0}$ or to several voxels (i.e. a region of interest). We will formulate everything in terms of $K$ since considering the distance to a larger subset of $\mathcal{M}$ can be interesting.

\subsection{Distance on Riemannian Manifolds}

Let us now further discuss the notion of distance function on a Riemannian manifold. Given two points $x, y \in \mathcal{M}$, we consider all the piecewise differentiable curves joining $x$ to $y$. For a Riemannian manifold, such curves do exist and we have the

Definition 3.1.1. The distance $\phi(x, y)$ is defined as the infinimum of the lengths of the $\mathcal{C}^{1}$ curves starting at $x$ and ending at $y$.

The length $\mathcal{L}$ of a curve $\gamma$ joining the two endpoints $x=\gamma\left(t_{1}\right)$ and $y=\gamma\left(t_{2}\right)$ is defined by:

$$
\mathcal{L}(x, y)=\int_{t_{1}}^{t_{2}}|\dot{\gamma}(t)|_{\gamma(t)} d t=\int_{t_{1}}^{t_{2}} \sqrt{\langle\dot{\gamma}(t), \dot{\gamma}(t)\rangle_{\gamma(t)}} d t .
$$

Moreover, we also have (Mantegazza and Mennucci (2002)) the

Proposition 3.1.1. If $x_{0} \in \mathcal{M}$, the function $u: \mathcal{M} \rightarrow \mathbb{R}$, called distance function and given by $u(x)=\phi\left(x, x_{0}\right)$, is continuous on $\mathcal{M}$ but in general it is not everywhere differentiable.

We adopt the notation $u(x)$ for the clarity of expressions but recall that $u$ depends of course on $x_{0}$. We consider a general Hamilton-Jacobi partial differential equation with Dirichlet boundary conditions

$$
\begin{cases}H(x, \nabla u(x))=0 & \text { on } \mathcal{M} \backslash K \\ u(x)=u_{0}(x) & \text { when } x \in K\end{cases}
$$

where $u_{0}$ is a continuous real function on $K$ and the Hamiltonian $H: \mathcal{M} \times T^{\star} \mathcal{M} \rightarrow \mathbb{R}$ is a continuous real function. We make the assumption that $H(x,$.$) is convex and we set$ $u_{0}(x)=0 \forall x \in K$.

We denote by $|v|$ the magnitude of a tangent vector $v$, defined as $\sqrt{g(v, v)}$. In matrix notation, by forming $G=\left\{g_{i j}\right\}$ the metric tensor, this writes $\sqrt{v^{T} G v}$. Then, by setting $H(x, p)=|p|-1$, we will work on the following theorem (for details on viscosity solutions on a Riemannian manifold, we refer to Mantegazza and Mennucci (2002)) 
Theorem 3.1.1. The distance function $u$ is the unique viscosity solution of the HamiltonJacobi problem

$$
\begin{cases}|\operatorname{grad} u|=1 & \text { on } \mathcal{M} \backslash K \\ u(x)=0 \quad \text { when } x \in K\end{cases}
$$

in the class of bounded uniformly continuous functions.

This is the well-known Eikonal equation on the Riemannian manifold $\mathcal{M}$. The viscosity solution $u$ at $x \in \mathcal{M}$ is the minimum time $t \geq 0$ for any curve $\gamma$ to reach a point $\gamma(t) \in K$ starting at $x$ with the conditions $\gamma(0)=x$ and $\left|\frac{d \gamma}{d t}\right| \leq 1 . u$ is the value function of the minimum arrival time problem. This will enable us to solve equation 4 as a dynamic problem and thus to take advantage of the great flexibility of level set methods. On the basis of Osher (1993), Sethian (1996a), Tsai et al. (2003b) and Chen et al. (1991), we reformulate equation 4 by considering $u$ as the zero level set of a function $\psi$ and requiring that the evolution of $\psi$ generates $u$ so that

$$
\{\psi(x, t)=0\} \Leftrightarrow\{u(x)=t\}
$$

Osher (1993) showed by using Theorem 5.2 from Chen et al. (1991) that, under the hypothesis that the Hamiltonian $H$ is independent of $u$, the level set generated by 5 is a viscosity solution of 4 if $\psi$ is the viscosity solution of

$$
\left\{\begin{array}{l}
\frac{\partial \psi}{\partial t}+F(t, x, \nabla \psi(t, x))=0 \quad \forall t>0 \\
\psi(x, 0)=\psi_{0}(x)
\end{array}\right.
$$

provided that the speed $F$ is strictly positive and does not change sign. This is typically the case for our anisotropic Eikonal equation where

$$
F(t, x, \nabla \psi)=H(t, x, \nabla \psi)+1=|\operatorname{grad} \psi|
$$

To find our solution, all we need to do is thus to evolve $\psi(x, t)$ while tracking, for all $x \in \Omega$, the time $\bar{t}$ when it changes sign. Now we have to solve 6 with $F(t, x, \nabla \psi)=|\operatorname{grad} \psi|$. We recall that for any function $f \in \mathbb{F}$, where $\mathbb{F}$ denotes the ring of smooth functions on $\mathcal{M}$, the metric tensor $G$ and its inverse define isomorphisms between tangent vectors (in $T \mathcal{M}$ ) and 1-forms (in $\left.T^{\star} \mathcal{M}\right)$. In particular, we have seen earlier that the gradient operator is defined as 
$\operatorname{grad} f=G^{-1} \nabla f$ where $\nabla f$ denotes the first-order differential of $f$. It directly follows that

$$
|\operatorname{grad} \psi|=\sqrt{g(\operatorname{grad} \psi, \operatorname{grad} \psi)}=\left(g_{i j} \frac{\partial \psi}{\partial \mathbf{x}^{l}} g^{l i} \frac{\partial \psi}{\partial \mathbf{x}^{k}} g^{k j}\right)^{1 / 2}=\left(\frac{\partial \psi}{\partial \mathbf{x}^{k}} \frac{\partial \psi}{\partial \mathbf{x}^{l}} g^{k l}\right)^{1 / 2}
$$

and we now present the numerical schemes used to estimate geodesics as well as the viscosity solution of

$$
\psi_{t}+|\operatorname{grad} \psi|=0
$$

\subsection{Numerical Schemes}

Numerical approximation of the hyperbolic term in 8 is now carefully reviewed on the well-known basis of available schemes for hyperbolic conservative laws. We seek a threedimensional numerical flux approximating the continuous flux, $|\operatorname{grad} \psi|^{2}$ and that is consistent and monotonic so that it satisfies the usual jump and entropy conditions and converges towards the unique viscosity solution of interest. References can be found in LeVeque (1992). On the basis of the Engquist-Osher flux in Sethian (1996a) and the approach by Kimmel et al. (1995) for level set distance computation on 2D manifolds, we propose the following numerical flux for our quadratic Hamiltonian $\nabla \psi^{T} G^{-1} \nabla \psi$ :

$$
\begin{gathered}
|\operatorname{grad} \psi|^{2}=\sum_{i=1}^{3} g^{i i}\left(\max \left(\nabla_{\mathbf{x}^{i}}^{-} \psi, 0\right)^{2}+\min \left(\nabla_{\mathbf{x}^{i}}^{+} \psi, 0\right)^{2}\right)+ \\
\sum_{\substack{i, j=1 \\
i \neq j}}^{3} g^{i j} \operatorname{minmod}\left(\nabla_{\mathbf{x}^{i}}^{+} \psi, \nabla_{\mathbf{x}^{i}}^{-} \psi\right) \operatorname{minmod}\left(\nabla_{\mathbf{x}^{j}}^{+} \psi, \nabla_{\mathbf{x}^{j}}^{-} \psi\right)
\end{gathered}
$$

where the $\nabla_{\mathbf{x}^{i}}^{ \pm} \psi$ are the forward/backward approximations of the gradient in $\mathbf{x}_{i}$, and the minmod operator is a flux limiter that ensures the stability of the numerical scheme. When the two arguments have the same sign, it returns the one with the smallest absolute value. When the two arguments have opposite signs, it returns zero. First of all, we point out that, because of equation 7 , we only have to use the inverse of the metric, ie. the diffusion tensor, and not the metric itself. Consequently, we do not have to invert the diffusion tensor with this approach, which is attractive from a numerical standpoint. However, as we will see in the next section, we will have to invert the tensor with the stationary formulation of the anisotropic Eikonal equation and this may be a source of numerical instability if tensors 
are estimated with the classical least-squares approach. In all the numerical experiments of this paper, we will rely on the framework described in Lenglet et al. (2006) to estimate diffusion tensors or generate Gaussian noise in the tensor space that respects the properties of those symmetric positive-definite matrices, so we can not end up with degenerate tensors. We have also experimented with higher order approximation schemes in order to increase the accuracy of the method. This is done by introducing WENO schemes in our numerical flux instead of the upwind gradients. WENO schemes are based on ENO (essentially nonoscillatory) schemes, which were first introduced by Harten et al. (1987) in the form of cell averages. They use a polynomial approximation of the derivatives and avoid oscillations when a shock is detected. WENO schemes of Liu et al. (1994), instead of approximating the numerical flux using the best candidate stencil, use a weighted convex combination of all the candidates stencils. We now quickly describe a very well-known method that speeds up the estimation of the distance function $u$.

Narrow Band Method In order to overcome the high computational overhead of the front propagation approach $\left(\mathcal{O}\left(N^{3}\right)\right.$ if $N$ denotes the number of grid points along a side), the narrow band method relies on the fact that it is sufficient to compute the level set function only in a small neighborhood (at a distance $\delta$ ) around its zero level set because only this zero level set is physically meaningful (representing the interface). It was introduced by Chopp (1993). In that case, the complexity drops to $\mathcal{O}\left(k N^{2}\right)$ in three dimensions, where $k$ is the number of voxels in the band. In practice, at an iteration $n$, only the points in the band are updated and other points are kept intact. When the front moves near to the edge of the band, the calculation is stopped and a new band is initialized with the zero level set interface boundary at the center.

We finally describe the method used for the computation of geodesics, in order to approximate paths of diffusion on $\mathcal{M}$ eventually corresponding to neural fibers tracts. Geodesics are classically obtained by performing back-propagation from a given point $x$ to the source $x_{0}$, in the direction provided by the opposite of the distance function gradient. Our 
problem of interest thus consists in starting from a given voxel of the white matter and in computing the optimal pathway in term of the distance $u$ until $x_{0}$ is reached. The simplest way to infer a geodesic path from $u$ is to backtrace on the manifold itself by solving the ordinary differential equation

$$
\frac{d \gamma(t)}{d t}=-\operatorname{grad} u=-G^{-1} \nabla u
$$

where $\gamma$ is the sought geodesic parameterized by $t$. $\operatorname{grad} u$ involves the Riemannian metric as well as the differential of the distance function $\nabla u$, evaluated with appropriate finite difference schemes. We have experimented with Euler, $2^{\text {nd }}$ and $4^{\text {th }}$ order Runge-Kutta integration methods with sensibly better results obtained by higher order Runge-Kutta schemes but noticeable computational overheads. When integrating within a voxel, trilinear interpolation of the vector field grad $u$ is performed by using the 8 available values in the neighborhood (ie. the corners of the voxel of interest).

While a given geodesic is estimated, we also compute on-the-fly statistics of a confidence measure $\mathcal{C}$ along this curve. Typically, as proposed in Parker et al. (2002b) we take $\mathcal{C}$ to be the absolute value of the Euclidean inner product between the normalized geodesic velocity and the principal diffusion vector of the interpolated diffusion tensor field at location $\gamma(t)$ :

$$
\left|\left\langle\frac{\dot{\gamma}(t)}{|\dot{\gamma}(t)|_{E}}, \mathbf{u}^{1}\right\rangle\right|
$$

This quantity reflects how well the local orientation of the geodesic matches the directional information provided by the diffusion tensor image. In practice, we can use the minimum of this quantity along $\gamma$ as a "worst case" value (as close to 1 as possible), its average (as close to 1 as possible) and standard deviation (as low as possible) in order to discriminate geodesics that are likely to represent true neural bundles from false connections.

Numerical experiments for the distance function computation and the geodesics estimation will be presented in section 6. An application of this method to quantify the likelihood of connection between the putamen and the motor areas of the human brain are 
presented in Lehericy et al. (2005). As we will see in the following, the method that was just described, though quite easy to implement, presents some drawbacks in terms of efficiency and robustness and they will be detailed in section 6 . We will now introduce the counterpart of the dynamic formulation of the Eikonal equation, namely its stationary formulation. This approach is based on notions from control theory, which we introduce in the next section before proposing the associated algorithm.

\section{From Geometry to Control Theory}

As in the previous section and in Wedeen et al. (1995), O'Donnell et al. (2002), Lenglet et al. (2004), the white matter is interpreted as a Riemannian manifold and the inverse of the diffusion tensor $D_{x}^{-1}$, at location $x$, provides the Riemannian metric, which in turn approximates white matter fibers as geodesic paths.

\subsection{Overview}

We remind the basic definition of geodesics for convenience (see Do Carmo (1992) for more details) and to clarify the notations.

Definition 4.1.1. Let $\mathcal{M}$ be a Riemannian manifold. Let $x, y \in \mathcal{M}$. The geodesic connecting $x$ to $y$ is the curve $\gamma_{0}$ which minimizes the arc length, i.e.

$$
\gamma_{0}=\arg \min _{\gamma \in \Gamma x, y} \int_{0}^{T_{x y}}\left|\gamma^{\prime}(t)\right|_{R} d t
$$

where $\Gamma x, y$ is the set of curves $\gamma:\left[0, T_{x y}\right] \rightarrow \mathcal{M}$ such that $\gamma(0)=x, \gamma\left(T_{x y}\right)=y$ and $\left|\gamma^{\prime}(t)\right|_{R}=1$, with $|\cdot|_{R}$ the norm associated with the Riemannian metric.

In section 2, we showed that the appropriate metric to our problem is the one associated with the norm $|\cdot|_{R}$ defined by $|x|_{R}=\sqrt{x^{T} D_{x}^{-1} x}$, where $D_{x}$ is the symmetric positive definite $3 \times 3$-matrix given by the measured diffusion tensor. Let us also denote by $A_{x}$ the (symmetric positive definite) square root matrix of $D_{x}$ and with $|\cdot|_{E}$ the Euclidean norm. Let us note that we trivially have

$$
|x|_{R}=\left|A_{x}^{-1} x\right|_{E}
$$


Here, rather than interpreting the problem in terms of Riemannian geometry, we adopt an optimal control point of view. The two interpretations are equivalent, but focus on different aspects of the problem. In the Riemannian setting, the emphasis is on the description of the geometry and in particular on the geodesics. In the optimal control interpretation, the emphasis is on the optimal control which coincides here with the intrinsic gradient of the distance function and with the vector field tangent to the geodesics. Also, from the computational point of view, the intrinsic gradients are much more important and relevant than the geodesics by themself (see next sections). In particular, the intrinsic gradients are fundamental for numerically estimating the connectivity measure. Moreover the geodesics can be directly computed from them.

Another advantage of the optimal control interpretation is the relaxation of the dependency with respect to the specific geometry of the manifold. In the control interpretation, all the objects are governed by the Euclidean metric.

Finally, the white matter is an open subset of $\mathbb{R}^{3}$. If one forsakes the specific metric attached to the white matter, that is to say, if one interprets the metric in another way (i.e. not as a metric), then the representation of the white matter as a manifold is unnecessary. Also, in the optimal control interpretation, we do not need to deal with manifold (see Venkataraman et al. (2002), Iyer et al. (2006)). We will come back to the advantages of this interpretation at the end of this section.

\subsection{Optimal Control Problems}

In this paragraph, we briefly state the determinist continuous optimal control problems on open subset of $\mathbb{R}^{n}$, as in Bardi and Capuzzo-Dolcetta (1997). We also describe the links between the introduced mathematical objects and the brain connectivity mapping problem.

The problem is the following: We want a particle to reach a certain target. We can control the trajectory of the particle thanks to a "control". The problem consists then in finding the optimal sequence of controls which minimizes a certain cost among all the sequences for which the particle reaches the target. This is how we obtain the optimal trajectory for the particle. 
In comparison with Riemannian geometry, this optimal trajectory plays the same role as a geodesic. Nevertheless, we are not directly interested in the trajectory but more in the control, which is a more subtle mathematical object and which provides various interesting tools. Let a domain $\Omega$ be a subset of $\mathbb{R}^{n} . \Omega$ is the space in which the particle can move. We consider a target $\mathcal{T} \subset \Omega$. Specifically in our problem, the domain $\Omega \subset \mathbb{R}^{3}$ represents the white matter; the target $\mathcal{T}$ is the point of interest $x_{0}$, origin of the distance function.

We consider the set $\mathcal{A}$ (compact subset of $\mathbb{R}^{m}$ ) of admissible controls $a(a \in \mathcal{A})$. We call control function, a function $\alpha():. \Omega \rightarrow \mathcal{A}$. In the following, the functions $\xi():. \mathbb{R}^{+} \rightarrow \Omega: t \mapsto \xi(t)$ are candidate trajectories for the particle. The control functions $\alpha($.$) control the dynamics of the$ trajectories of the particles. Mathematically, this control is represented as a vector field

$$
f: \Omega \times \mathcal{A} \rightarrow \mathbb{R}^{n}:(x, a) \mapsto f(x, a)
$$

and we pose

$$
\xi^{\prime}(t)=f(\xi(t), \alpha(\xi(t))), t>0
$$

In other words, we control the trajectory of the particle by adapting its velocity vector (its derivative) via the control "parameter" $\alpha$, which we can optimize at any time. $f$ is called the dynamics. Under some regularity assumptions, to each control function $\alpha$ and $x \in \Omega$, we can associate a single trajectory $\xi_{x, \alpha}(t) \in \Omega$ following the dynamics $\xi^{\prime}(t)=f(\xi(t), \alpha(\xi(t))), t>0$ and s.t. $\xi(0)=x$, imposed by the control $\alpha$ (Bardi and Capuzzo-Dolcetta (1997)).

Now, let us define a (local) cost

$$
l: \Omega \times \mathcal{A} \rightarrow \mathbb{R}:(x, a) \mapsto l(x, a) .
$$

To each control function $\alpha$ and $x \in \Omega$ (which then determines a trajectory), we can then associate a global cost: the integral of the (local) cost along the associated trajectory $\xi_{x, \alpha}($.$) ,$ i.e.

$$
\int_{0}^{T_{x, T, \alpha}} l\left(\xi_{x, \alpha}(t), \alpha\left(\xi_{x, \alpha}(t)\right)\right) d t
$$

where $T_{x, \mathcal{T}, \alpha}$ is the first time for which the trajectory $\xi_{x, \alpha}$ (controlled by the dynamics $f$ ) reaches the target $\mathcal{T}$. Under some regularity assumptions, one can prove that there exists a control function $\alpha^{*}$ (the optimal control) such that for all $x$, the global cost (11) is minimal 
(Bardi and Capuzzo-Dolcetta (1997)):

$$
\alpha^{*} \stackrel{\text { def }}{=} \arg \min _{\alpha} \int_{0}^{T_{x, \tau, \alpha}} l\left(\xi_{x, \alpha}(t), \alpha\left(\xi_{x, \alpha}(t)\right)\right) d t
$$

We then denote the optimal trajectory starting from $x$ by

$$
\xi_{x}^{*} \stackrel{\text { def }}{=} \xi_{x, \alpha^{*}}
$$

and the optimal dynamics at $x$ by

$$
f_{x}^{*} \stackrel{\text { def }}{=} f\left(x, \alpha^{*}(x)\right)
$$

In our specific problem, we claim that the optimal trajectories must be in close agreement with the white matter fibers, if the start point $x$ and the target point $x_{0}$ of the white matter are indeed connected by a fiber (If they are not, the connectivity measures presented in section 4.5 should be able to discard such false-positive connections). The optimal controls and optimal dynamics give the direction of the fibers; the other candidate trajectories and other control values describe the spectrum of all the possible curves connecting $x$ to $x_{0}$, while staying within the white matter. In the optimal control problems, the goal is then to characterize and compute this optimal control $\alpha^{*}$ which minimizes the cost (11) $\left(f_{x}^{*}\right.$ being immediately deduced from $\alpha^{*}$ ).

\subsection{Geodesics and the Optimal Control Framework}

In this section, we show how one can rigorously make the link the between Riemannian geometry and optimal control theory. More precisely, we design a specific control problem for which the optimal trajectories coincide with the Riemannian geodesics considered in Lenglet et al. (2004).

Now, let us consider the above optimal control problem with

$$
l(x, a)=1 .
$$

The problem consists in finding the control function $\alpha^{*}$ s.t. for all $x$ in $\Omega$ and for all $\alpha$

$$
\int_{0}^{T_{x, x_{0}, \alpha^{*}}} l\left(\xi_{x, \alpha^{*}}(t), \alpha^{*}\left(\xi_{x, \alpha^{*}}(t)\right)\right) d t \leq \int_{0}^{T_{x, x_{0}, \alpha}} l\left(\xi_{x, \alpha}(t), \alpha\left(\xi_{x, \alpha}(t)\right)\right) d t, \quad \text { s.t. }
$$




$$
T_{x, x_{0}, \alpha^{*}} \leq T_{x, x_{0}, \alpha}
$$

and where $T_{x, x_{0}, \alpha}$ is the first time for which the trajectory $\xi_{x, \alpha}$ (controlled by the dynamics $f$ ) reaches the target $x_{0}$. For simplicity, we have fixed $\mathcal{T}=\left\{x_{0}\right\}$ and we have $T_{x, x_{0}, \alpha}=+\infty$ if the trajectory does not reach $x_{0}$. In other words, misusing the notations ( $\alpha^{*}$ minimizes (13) for all $x \in \Omega$ and we can prove that $\alpha^{*}$ always exists, see Bardi and Capuzzo-Dolcetta (1997)), $\alpha^{*}$ is

$$
\alpha^{*}=\arg \min _{\alpha(.)}\left\{\int_{0}^{T_{x, x_{0}, \alpha}} 1 d t\right\}=\arg \min _{\alpha(\cdot)}\left\{T_{x, x_{0}, \alpha}\right\}
$$

Furthermore let $\mathcal{A}$ be the unit Riemannian sphere $\mathcal{S}_{R}(0,1)$ associated with $A_{x}$, i.e.

$$
\mathcal{A}=\mathcal{S}_{R}(0,1)=\left\{A_{x}^{T} b \mid b \in \mathcal{S}_{E}(0,1)\right\},
$$

$\mathcal{S}_{E}(0,1)$ being the Euclidean unit sphere, and let

$$
f(x, a)=-a
$$

i.e. the dynamics is equal to the control (up to the sign). Then for all $t, \xi^{\prime}(t)=-\alpha(\xi(t))$ covers the unit Riemannian sphere when $\alpha$ covers the set of the functions $\Omega \rightarrow \mathcal{A}$.

Let us denote $T_{\xi, \mathcal{T}}$ the first time $t$ for which the trajectory $\xi$ reaches the target $\mathcal{T}$, i.e. for which $\xi(t) \in \mathcal{T}$. We have then $T_{x, x_{0}, \alpha}=T_{\xi_{x, \alpha},\left\{x_{0}\right\}}$. So, minimizing the cost

$$
\int_{0}^{T_{x, x_{0}, \alpha}} 1 d t
$$

for $\alpha: \Omega \rightarrow \mathcal{A}$ is equivalent to minimize

$$
\int_{0}^{T_{\xi, x_{0}}} 1 d t
$$

for $\xi$ such that $\left|\xi^{\prime}(t)\right|_{R}=1$. In other words, in this case, the optimal trajectories $\xi_{x}^{*}$ correspond with the geodesics considered in Lenglet et al. (2004) (see Definition 4.1.1). Also, in the following, instead of dealing with geodesics, we are going to recover optimal trajectories corresponding to this specified optimal control problem and we are going to take advantage of the benefits of the control theory.

\subsection{Advantages of the Optimal Control Interpretation}

The control interpretation has distinct advantages: All the objects of interest live in $\mathbb{R}^{3}$ (instead of a manifold), and are governed by the Euclidean metric, hence the interpretation 
is independent of the geometric structure. As an illustration of this benefit, in order to estimate the direction of the geodesics, we proposed in section 3 to compute the gradient of the distance function on the manifold, which requires some care in order to take into account the geometry imposed by the metric and is a challenging task when working on an irregular domain such as the brain white matter. In the control formalism the interpretation is rather direct: the tangent of the geodesics is in fact the optimal dynamics $f_{x}^{*}$ (since the geodesic corresponds to the optimal trajectories). Also, the optimal dynamics $f_{x}^{*}$ coincides with the optimal control (up to its sign), which is the direct outcome of our algorithm.

The control framework reveals the fact that the value function $V$ defined by the min of (11)

$$
V(x)=\min _{\alpha}\left\{\int_{0}^{T_{x, x_{0}, \alpha}} l(\xi(t), \alpha(\xi(t))) d t\right\}
$$

is the viscosity solution (Lions (1982), Bardi and Capuzzo-Dolcetta (1997)) of the partial differential equation $(\mathrm{PDE})$

$$
\sup _{a \in \mathcal{A}}\{-f(x, a) \cdot \nabla u(x)-l(x, a)\}=0,
$$

verifying $u\left(x_{0}\right)=0$ and the state constraints on the boundary of the domain $\partial \Omega$ (Soner (1986), Prados et al. (2006c)). This result is quite general and it applies to any optimal control problem as the one described in section 4.2. In our specific case, for $f(x, a)=-a, l(x, a)=1$ and $\mathcal{A}=\mathcal{S}_{R}$ (see section 4.3), equation (15) coincides with the explicit equations:

$$
\left|A_{x} \nabla u\right|_{E}=1
$$

i.e.

$$
|\nabla u|_{R}=1 .
$$

Also, we logically associate with this PDE the explicit Hamiltonian

$$
H_{A E i k}(x, p)=\left|A_{x} p\right|_{E}-1=|p|_{R}-1 .
$$

Proof. By the Cauchy-Schwarz inequality, we have

$$
\left|A_{x} p\right|_{E}=\sup _{b \in \mathcal{S}_{E}(0,1)}\left(A_{x} p\right) \cdot b=\sup _{b \in \mathcal{S}_{E}(0,1)} p \cdot\left(A_{x}^{T} b\right) .
$$


Then $\left|A_{x} p\right|_{E}=\sup _{a \in \mathcal{S}_{R}(0,1)} p \cdot a$. So $\sup _{a \in \mathcal{S}_{R}(0,1)}\{a \cdot p-1\}=\left|A_{x} p\right|_{E}-1$.

In other respects, in our application, we have

$$
V(x)=\min _{\alpha}\left\{\int_{0}^{T_{x, x_{0}, \alpha}} 1 d t\right\}=\min _{\alpha} T_{x, x_{0}, \alpha} .
$$

Since the control values stay in the Riemannian unit sphere $S_{R}(0,1)$, we tautologically have

$$
|a|_{R}=1
$$

Since we have moreover

$$
|a|_{R}=|f(\xi(t), a)|_{R}=\left|\xi^{\prime}(t)\right|_{R},
$$

then all the candidate trajectories have a constant speed of norm 1 for the Riemannian metric. The covered distance coincides then with the time required for the trajectories to reach the target. This was at the basis of the dynamic formulation in section 3 . The value function $V(x)$ defined by equation (19) is then naturally the distance function considered in the Riemannian context. Thus, one recovers the fact that the (anisotropic) distance function is the viscosity solution of the anisotropic Eikonal equation (17). The control framework, as in Bardi and Capuzzo-Dolcetta (1997), also reveals that $f\left(x, \alpha_{x}^{*}\right)=-\nabla H(x, \nabla u(x))$ where $H$ is the Hamiltonian associated with the PDE (15) and $\nabla u$ is the gradient of its solution. This trick is quite useful and efficient in practice for computing the optimal dynamics.

More globally, let us remind that the Riemannian geometry focuses on the description of the geometry and in particular on the geodesics, when the main concern of the optimal control framework is the optimal control which here coincides with the optimal dynamics (up to its sign). Also, as we will see in the next sections, from the computational point of view, the notion of optimal dynamics is fundamental. In particular, this notion is essential for designing an efficient numerical scheme. In fact, the control formulation of the problem directly yields our numerical method, which we report in sections 5.2 and 5.3. Finally, the notion of optimal dynamics is also at the basis of our connectivity measure, from the theoretical point of view as well as from the computational point of view. For practical purposes, we will adopt either interpretation (Riemannian geometry versus optimal control) depending on the situation and exploit their complementary benefits. 


\subsection{Connectivity Measures}

We would like to clarify and improve the connectivity measure $\mathcal{C}$ introduced in section 3.2 . We start by pointing out that, for a fixed point $x_{0}$ and any point $x$, the geodesic $\gamma_{x}$ (associated with the metric given by the inverse of the tensor) connecting $x$ to $x_{0}$ always exists. If $x$ is connected to $x_{0}$ by a white matter fiber bundle then the associated geodesic $\gamma_{x}$ is a reasonable approximation of such a fiber bundle. However, for any $x$, the associated geodesic $\gamma_{x}$ does not necessarily coincide with a fiber. Also, in order to reach our goal (reconstruction of the white matter fibers), we need to be able to trace the geodesics and to evaluate if a point is potentially connected to $x_{0}$.

In this section, we propose a well-founded score to measure the expectation that a given geodesic represents the connection between a point $x$ and $x_{0}$ with a certain level of confidence. By computing statistical maps of this measure for all points $x$ in the brain, we can then determine which points are likely to be connected to $x_{0}$ and then trace the fibers. In section 5 we propose an original numerical scheme based on Fast Marching Methods (FMM) to efficiently compute these maps.

Let us fix a point of interest $x_{0} \in \bar{\Omega}$ and let us consider the PDE/control/Riemannian problem associated with DTI. In section 4 , we show that, $\forall x \in \Omega$, the optimal dynamics $f_{x}^{*}$ coincides with the derivatives of the geodesics $\gamma^{\prime}(t)$ at $x$ and that they are in the Riemannian unit ball $B_{R}(0,1)$ which is also the set $\left\{A_{x} q, q \in B_{E}(0,1)\right\}$.

So, for a fixed point $x$ (and a fixed tensor $D_{x}$ ), the larger the Euclidean norm of $f_{x}^{*}$, the more confident we are in the local direction of the geodesic. Following this idea, we then define a general (local) confidence measure:

$$
\mathcal{C}(x)=\sqrt{f_{x}^{* T} D_{x}^{\alpha} f_{x}^{*}}
$$

$\alpha$ being in $\mathbb{R}$. In addition to being intuitive, this measure inherits the robustness to noise of the optimal dynamics. It also exploits the full information provided by the diffusion tensor. Finally, it does not penalize any direction in case of isotropy. Let us now discuss the possible values of $\alpha$ : if $\alpha=-1$, we get $\mathcal{C}(x)=1, \forall x \in \bar{\Omega}$. This simply means that, when we use 
the Riemannian metric given by the inverse of the diffusion tensor, all the geodesics are equivalent. On the contrary, when $\alpha=0$, we have $\mathcal{C}(x)=\left|f_{x}^{*}\right|_{E}$ and we claim that it is a natural local measure of connectivity since this measures the speed of propagation at $x$. Finally, when $\alpha \rightarrow \infty$, this boils down to considering the alignment of the optimal dynamics with the local major eigenvector. This was used in section 3.2 and in Parker et al. (2002b) but it may be sensitive to isotropic areas where, by definition, the major eigenvector is undefined.

From this local connectivity measure, we can define global information from its statistics (mean and standard deviation) along the optimal trajectory:

$$
\begin{gathered}
\mu(x)=\langle\mathcal{C}(x)\rangle=\frac{1}{\tau_{x}^{*}} \int_{0}^{\tau_{x}^{*}} \mathcal{C}\left(\xi_{x}^{*}(t)\right) d t \\
\sigma(x)=\sqrt{\left\langle\mathcal{C}(x)^{2}\right\rangle-\langle\mathcal{C}(x)\rangle^{2}}
\end{gathered}
$$

where $\tau_{x}^{*}$ is the length of the optimal trajectory $\xi_{x}^{*}$. We should point out that, since $\left|\xi_{x}^{*}\right|_{R}=1$, this length (i.e. the geodesic distance between the curve endpoints $x_{0}$ and $x$ ) coincides with the arrival time $T_{x, x_{0}}$ introduced in section 4 . A point $x$ connected to $x_{0}$ by a white matter fiber will ideally have a large value for $\mu(x)$ and a small standard variation $\sigma(x)$. The choice of using the mean instead of just integrating along the trajectories allows the comparison of two points $x$ and $y$ which are located at different distance from $x_{0}$, i.e. s.t. $\tau_{x}^{*} \neq \tau_{y}^{*}$. Although the mean value of the connectivity may be sufficient to discriminate likely fibers, the variance of this quantity may also be of great help since an ideal fiber would exhibit a high coherence of $\mathcal{C}(x)$ along its trajectory.

Remark 1. To compute the optimal dynamics, we need to compute also the geodesic distance, which in fact is equal to $\tau_{x}^{*}$. In practice, we just need to compute

$$
\mathcal{R}(x)=\int_{0}^{\tau_{x}^{*}} \mathcal{C}\left(\xi_{x}^{*}(t)\right) d t
$$

and

$$
\mathcal{S}(x)=\int_{0}^{\tau_{x}^{*}} \mathcal{C}\left(\xi_{x}^{*}(t)\right)^{2} d t
$$

The values of $\mu(x)$ and $\sigma(x)$ are then derived immediately by using the value $\tau_{x}^{*}$. 


\section{A Fast Numerical Algorithm}

We now describe an algorithm which can, in one single pass over the volume $\Omega$, compute all the quantities of interest.

\subsection{Related Work and Contributions}

To the best of our knowledge, except Melonakos et al. (2007), Pichon et al. (2005), there is no algorithm to compute directly the geodesics or a fiber connectivity confidence map to a point $x$ from DTI data. All the methods recovering white matter fibers by using some front propagation technique proceed by implementing successively the following four steps:

1. Computation of the distance function to $x$;

2. Extraction of the gradients of the distance function;

3. Estimation of the optimal dynamics from the gradients of the distance function;

4. Tracing of the geodesics from the computed directions. This step needs in particular an interpolation of the derivatives of the geodesics.

Some slight variants are proposed in the literature (see Cohen (2005), Kimmel and Sethian (1998) and references therein). For example, in the particular case of the isotropic Eikonal equation (where the optimal dynamics coincide with the gradient of the distance function), Kimmel and Sethian (1998) suggest not to compute the gradients for all voxels and later interpolate them, but rather to directly compute the interpolated gradients from the distance function.

We wish to emphasize that the explicit tracing of the geodesics is a prerequisite to all the previous methods for computing connectivity confidence measures which in fact consist in the integration of a local criterion along the entire geodesic during the geodesics tracing step. Thus, the estimation of a complete map of connectivity measures needs to explicitly trace all the geodesics starting from all the points of the map. This approach is rather computationally intensive. 
The numerical method we propose here for computing the confidence measures does not need to trace any geodesic. The confidence measure map is a direct output of our algorithm. It simultaneously and consistently computes the (geodesic) distance function, the optimal dynamics and the confidence measures.

The methods of the type "Fast Marching" (see Tsitsiklis (1995), Sethian (1996b), Sethian and Vladimirsky (2003), Prados and Soatto (2005)) are "one-pass" methods allowing to solve numerically partial differential equations of the type (15). Based on a causality principle, the Fast Marching Methods (FMM) stand in contrast to iterative methods (see for example Rouy and Tourin (1992), Tsai et al. (2003a) and more specifically the Fast Sweeping Method used in Jackowski et al. (2005)) which iteratively update the approximation of the solution by using paths that do not depend on the data. The idea of the FMM consists in computing the solution of the PDE as a front propagates along the optimal trajectories. Moreover, it has been recently shown in Yatziv et al. (2006) that using a quantization of the priorities in the marching computation reduces the original complexity of FMM from $\mathcal{O}(N \log N)$ to $\mathcal{O}(N)$.

Iterative methods (Pichon et al. (2005), Melonakos et al. (2007), Fletcher et al. (2007), Jackowski et al. (2005)) as well as Fast Marching Method (Jbabdi et al. (2008), Parker et al. (2002b)) have already been used in tractography applications for which one needs to compute the geodesic distance. A recursive Fast Marching Method has also been recently proposed and used by Konukoglu et al. (2007). Our algorithm extends the previous FMM by computing and returning, in addition, the optimal dynamics and connectivity confidence measures. The consistency of our results relies on the fact that for all the computations we use the same (optimal) simplex.

Remark 2. 1) All the quantities we compute are essential: The optimal dynamics is 
necessary in order to trace the geodesics, which in turn is useful for the visualization of the fibers. Even if the result of the computation of the (geodesic) distance is not required for tracing the geodesic, it is essential for obtaining the final measures (expectation and standard deviation) we use in practice to estimate the connectivity confidence.

2) Our method is a "one pass method" based on front propagation. An important consequence is that we do not need to wait for the complete computation of the distance function on the whole domain to be able to exploit it for computing the connectivity measures. Also, if at any time the process stops, all the values already computed are valid approximations, unlike in other iterative techniques.

Remark 3. The basic (anisotropic) Fast Marching algorithm does not systematically approximate well the continuous solution of an anisotropic Eikonal equation (the quality of the reconstruction depends on the anisotropy of the metric but also on the direction of the eigenvectors with respects to the mesh structure). There are mainly three ways to improve the numerical solutions computed by methods of type Fast Marching: 1) One can change the approximation scheme, 2) One can introduce iterative procedures (as it is done by Konukoglu et al. (2007)), 3) One can extend the neighborhoods (see Sethian and Vladimirsky (2003) and Jbabdi et al. (2008)). From a theoretical point of view, the convergence of the computed solution toward the continuous solution (when the mesh vanishes) has only been proved for the fully iterative algorithms (theory developed by Barles and Souganidis) and for the OUM (i.e. with extended neighborhoods) with a semi-Lagragian scheme, see Sethian and Vladimirsky (2003). In all other cases, the convergence toward the continuous solutions is not proved and, in most of cases, probably does not hold. A difficulty in our application is that the anisotropy is substantial; also to satisfy the theoretical conditions ensuring the convergence of the OUM, we would need extremely vast neighborhoods (typically a sphere with a radius of 5 to 10 voxels). This neighborhood must be updated for each progression of the front (i.e. each time a voxel is transferred from the narrow band to the set of known points). Moreover in our problem, we have to handle the convoluted topology of the white matter (see section 6.2 .1 ) and 
accordingly manage the configuration of neighborhoods. This can be complicated and pose several numerical problems and implementation difficulties. Since in practice, given the poor spatial resolution of DTI, the extension of the neighborhood does not result in significant differences for the computed solution, it appears that large neighborhoods, as recommended by the theory, is not necessarily relevant to our problem. We have chosen to use a 6-neighborhood (see section5.3.1), but the 26-neighborhood recently proposed by Jbabdi et al. (2008) also appears to be a good alternative.

Remark 4. The control formulation used by Pichon et al. (2005), Melonakos et al. (2007) is similar to ours. Nevertheless they do not exploit this formulation as we do since they use it to design an iterative sweeping algorithm with a semi-Lagrangian scheme. Moreover, Pichon et al. and Melonakos et al. compute the distance function and the optimal dynamics and, from these quantities, trace geodesics (Pichon et al. (2005)) or segment fiber bundles (Melonakos et al. (2007)) between two points of interest (a starting and target point). They do not compute connectivity measures as we do. It is clear that such iterative schemes do not allow to recover efficiently and simultaneously the distance function, optimal dynamics and connectivity measures. The computation of the connectivity measures requires the availability of the optimal dynamics. Consequently, Pichon et al. (2005), Melonakos et al. (2007) can only estimate these quantities upon convergence of their iterative procedure. In other words, with such iterative methods, the connectivity measures would have to be computed in a second step, after having fully computed the distance function and the optimal dynamics. In Fletcher et al. (2007), the authors do not really interpret the problem in terms of control. The framework they use corresponds more to the one of the Riemannian theory. In fact, the main contribution of this work relies on the introduction of a new method to recover a volumetric representation of the connectivity between two regions by using the sum of the two distance functions to each region.

In the following, we describe our global algorithm and then the implementation of each specific step. 


\subsection{Global Algorithm}

As in the classical Fast Marching method (Sethian (1996b), Sethian and Vladimirsky (2003), Prados and Soatto (2005)), the grid points are divided into the three classes: Accepted, Considered, Far. Below $U, f, R$ and $S$ are respectively the approximations of the (geodesic) distance function, the optimal dynamics $f_{x}^{*}, \mathcal{R}$ and $\mathcal{S}$ (defined in section 4.5). $x_{0}$ is the interest point. The algorithm is then the following (Algorithm 1):

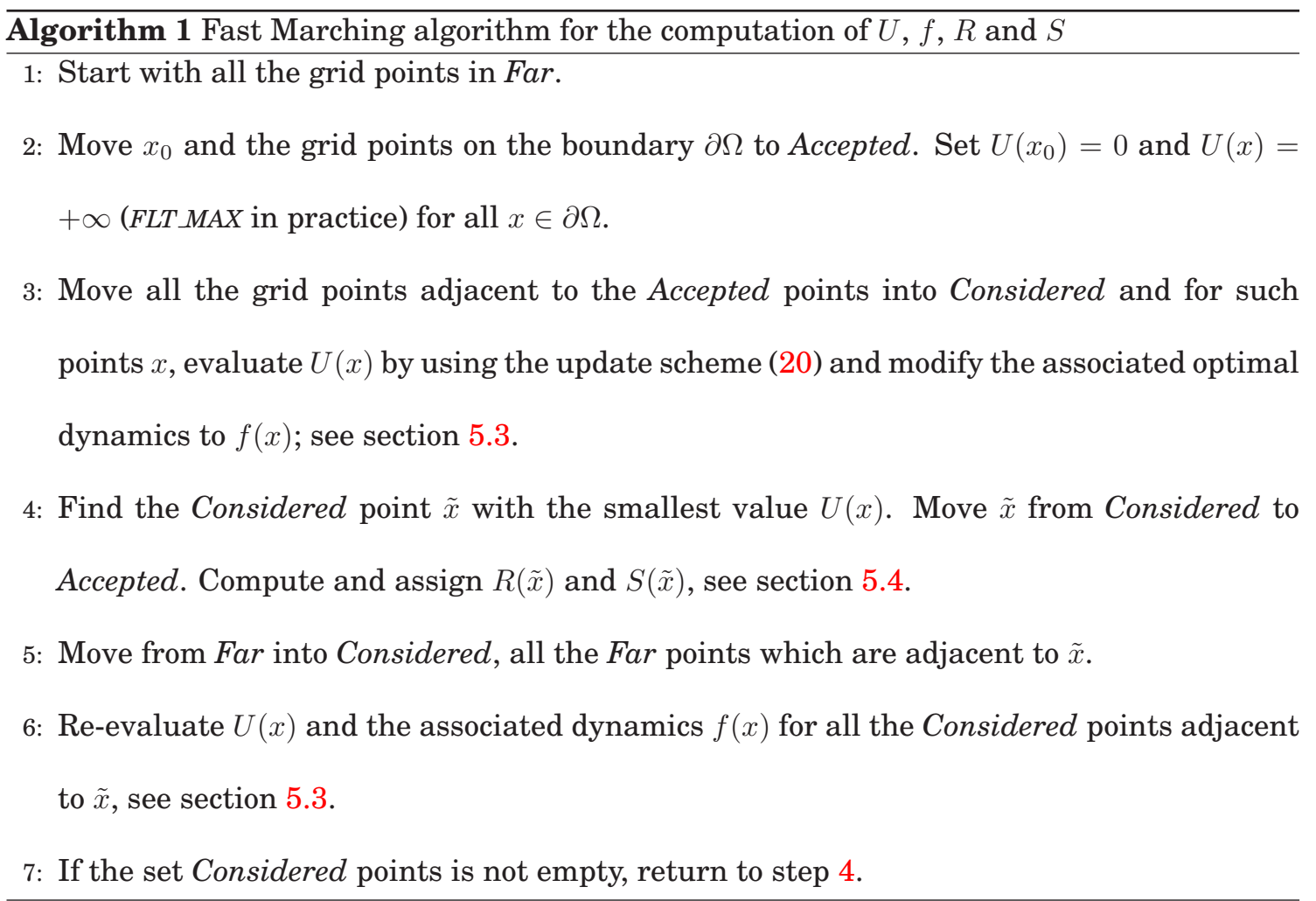

\subsection{Distance and Optimal Dynamics Computation}

Here, we focus on the implementation of the updating steps for the approximation of the distance function and the optimal dynamics (steps required in items 3 and 6 of Algorithm 1).

\subsubsection{Approximation Scheme}

The "approximation scheme" is the equation one has to solve in the updating step (steps 3 and 6 of Algorithm 1) to (re)compute the candidate value $U(x)$ at the voxel $x$ in the narrow band. This equation is of the form $S(\rho, x, t, U)=0$ where 
- $\rho$ corresponds to the size of the mesh. Here we have $\rho=\left(h_{1}, . ., h_{n}\right)$, where $h_{i}$ is the size of a voxel in the $i^{\text {th }}$ dimension; in our case, $n=3$.

- $x$ is the position of the considered voxel,

- $t \in \mathbb{R}$ is the unknown of the equation: $t$ is the candidate value we will affect to $U(x)$,

- $U$ contains the current values of $U$ (the geodesic distance function) in the neighborhood of $x$. Here, it is reduced to the values $U\left(x \pm h_{i} e_{i}\right)$, for all $i$ in $[1, . ., n]$.

Following Prados and Soatto (2005), we use the approximation scheme given by

$$
S(\rho, x, t, u)=\sup _{a \in A}\left\{-f(x, a) \cdot P_{s_{1}(x, a), . ., s_{n}(x, a)}-l(x, a)\right\}
$$

where $\left[P_{s_{1}, . ., s_{n}}\right]_{i}=\frac{t-U\left(x+s_{i} h_{i} e_{i}\right)}{-s_{i} h_{i}},\left\{e_{i}\right\}$ being the canonical basis of $\mathbb{R}^{n}$. In other words, since $t$ will be assigned to $U(x), P_{s_{1}, . ., s_{n}}$ is the approximation of the gradient obtained by using the values of $U$ on the simplex $\left(x, x+s_{1} h_{1} e_{1}, . ., x+s_{n} h_{n} e_{n}\right)$. In (20), we have denoted $s_{i}(x, a)=\operatorname{sign}\left(f_{i}(x, a)\right)$.

The scheme (20) is obtained by replacing $\nabla u$ by $P_{s_{1}, . ., s_{n}}$ in equation (15)

$$
\sup _{a \in \mathcal{A}}\{-f(x, a) \cdot \nabla u(x)-l(x, a)\}=0
$$

and by choosing the simplex, i.e. the signs $\left(s_{1}, . ., s_{n}\right)$, which contains the trajectory / dynamics of the associated control. In effect, for a fixed control $a \in A$, if $\overline{s_{i}}=\operatorname{sign}\left(f_{i}(x, a)\right)$, then the dynamics $f(x, a)$ is in the simplex defined by the points $\left(0, \overline{s_{1}} h_{1} e_{1}, . ., \overline{s_{n}} h_{n} e_{n}\right)$ and, for small enough $t$, the trajectory $\xi(t)=x+f(x, a) t+o(t)$ stays in the simplex $\left(x, x+\overline{s_{1}} h_{1} e_{1}, . ., x+\overline{s_{n}} h_{n} e_{n}\right)$.

This choice is also motivated to get a monotonic scheme (i.e. the function $u \mapsto S(\rho, x, t, u)$ is non increasing), which partly ensures that we will obtain an approximation of the viscosity solution of the considered PDE. One can easily verify that the scheme (20) is indeed monotonic (Prados and Soatto (2005)). We will take advantage of the various properties of that scheme to obtain simultaneously and consistently the approximations of the geodesic distance function and of the optimal dynamics. Moreover, we want to point out that this 
choice of simplex is consistent with those made in the classical upwind methods, for example in Sethian and Vladimirsky (2003).

Finally, let us emphasize that the scheme (20) is different from the schemes proposed in Tsitsiklis (1995), Sethian (1996b), Sethian and Vladimirsky (2003). The schemes proposed in Tsitsiklis (1995), Sethian (1996b), and also in Jbabdi et al. (2008) are semi-Lagrangian schemes. Eulerian schemes are proved to be more accurate and efficient than the semiLagrangian ones, in particular with the quadratic version of our equation (with a control of order 2), see for example Dupuis and Oliensis (1994), Sethian and Vladimirsky (2003), Qian et al. (2007). The scheme (20) is an Eulerian scheme and it is rather similar to the one proposed by Sethian and Vladimirsky (2003), section 8. Nevertheless, contrary to our scheme, the one proposed by Sethian and Vladimirsky is not a fully Eulerian scheme: it uses a semi-lagragian scheme for the "sided" cases, i.e. when the computed optimal dynamics are not inside the simplex but on its boundary (see section 8.2.2 of Sethian and Vladimirsky (2003)). The "sided" cases are really important because they are extremely frequent. Also, the effect of a poor management of these cases are significantly amplified with small neighborhoods. The scheme (20) is fully consistent with the anisotropic Eikonal equation (and in fact, more generally, with any Hamilton-Jacobi-Bellman equation). The proof of the consistency of the scheme (20) in the general case can be found in Prados (2004).

\subsubsection{Equation to solve}

The updating step consists in computing the value we want to assign to $U(x)$ from the values $U\left(x \pm s_{i} h_{i} e_{i}\right)$, and according to the chosen scheme. For a fixed voxel $x \in \Omega$, the update value for $U(x)$ is the solution of the equation in $t$

$$
S(\rho, x, t, U)=0
$$

Hence, the equation (in $t$ ) that we have to solve is

$$
\sup _{a \in A}\left\{-f(x, a) \cdot P_{s_{1}(x, a), \ldots, s_{n}(x, a)}-l(x, a)\right\}=0 .
$$


A natural way of solving an equation in $t$ of the form

$$
\sup _{a \in A} G(a, t)=0
$$

$G$ being a concave function, differentiable with respect to $a$, would be to explicitly rewrite this equation by using differential calculus, and then to solve the explicit equation in $t$. However, for equation (21), the associated function $G$ is not differentiable. It is only piecewise differentiable and we cannot rewrite it explicitly.

To solve equation (21), we are going to separate the problem into subproblems. Each subproblem will then consist in solving a "restricted" equation

$$
\sup _{a \in R} G(a, t)=0
$$

where the sup is restricted to the connected components $R$ on which $G$ is differentiable. The resolution of each "restricted" equation is easier than the global one since $G$ is differentiable and concave with respect to $a$ on each $R$. Also, among the various solutions $t_{R}$ of the "restricted" equations, we will select the solution of the global equation. In the next two sections, we are going to detail this process for the equation (21).

\subsubsection{Separation and choice of the optimal simplex}

First, let us rewrite equation (21) as

$$
\max _{s \in\{ \pm 1\}^{n}} \sup _{a \in A_{s}}\left\{-f(x, a) \cdot P_{x, s, U}(t)-l(x, a)\right\}=0
$$

where we note $s=\left(s_{1}, . . s_{n}\right) \in\{ \pm 1\}^{n},\left[P_{x, s, U}(t)\right]_{i}=\frac{t-U\left(x+s_{i} h_{i} e_{i}\right)}{-s_{i} h_{i}}$ and

$$
A_{s}=\left\{a \in A \mid \forall i=1 . . n, s_{i}(x, a)=s_{i}\right\} .
$$

Now, for all $s \in\{ \pm 1\}^{n}$, let us denote

$$
G_{s}(t)=\sup _{a \in A_{s}}\left\{-f(x, a) \cdot P_{x, s, U}(t)-l(x, a)\right\}
$$

and $t_{s}$, the solution of the equation (in $t$ )

$$
G_{s}(t)=0
$$


Since $G_{s}(t)$ is increasing with respect to $t$, the solution of $\max _{s} G_{s}(t)=0$ is $t_{0}=\min _{s \in\{ \pm 1\}^{n}} t_{s}$. Hence, the implementation of the update step is reduced to the computation of the $2^{n}$ solutions $t_{s}$ and, finally, to choosing the smallest one. We call optimal simplex the simplex associated to the smallest solution. In other respects, when we compute $t_{s}$, we also compute $f_{s}=f\left(x, a_{s}\right)$, where $a_{s} \in A_{s}$ is the optimal control of equation (22) for $t=t_{s}$. We can then associate to $t_{0}$ the optimal dynamics $f_{0}=f_{s}$ where $s$ is the optimal simplex.

\subsubsection{Computation of $t_{s}$ and the associated dynamics}

Now let us set $s=\left(s_{1}, . . s_{n}\right) \in\{ \pm 1\}^{n}$. If we denote

$$
g_{s}(a, t)=-f(x, a) \cdot P_{x, s, U}(t)-l(x, a)
$$

then $t_{s}$ is the solution of

$$
\sup _{a \in A_{s}} g_{s}(a, t)=0 \text {. }
$$

It turns out that

Proposition 5.3.1. $t_{s}$ is unique and that it always exists.

See appendix A for a proof of proposition 5.3.1. By continuity of $f(x,),. A_{s}$ is a closed subset of $\mathbb{R}^{m}$. Let us remind that $a_{s}$ in $A_{s}$ is the optimal control of equation (23) for $t=t_{s}$. We then have two cases:

1. $\forall k \in[1 . . n], f_{k}\left(x, a_{s}\right) \neq 0$ (in other words $a_{s} \in \operatorname{Interior}\left(A_{s}\right)$ ): One can prove that this is equivalent to: $t_{s}$ is the solution of the equation $H\left(x, P_{x, s, U}(t)\right)=0$ with the associated optimal control in $A_{s}$ (i.e. the optimal control of $\sup _{a \in A}\left\{-f(x, a) \cdot P_{x, s, U}(t)-l(x, a)\right\}=0$, which can be anywhere in the whole set A).

Proof. In effect, by concavity of $g_{s}$ (due to the convexity of $f(x, a)$ and $l(x, a)$ ) with respect to $a$, we have $\sup _{a \in A_{s}} g_{s}(a, t)=\sup _{a \in A} g_{s}(a, t)$. The conclusion follows from the fact that $\sup _{a \in A_{s}} g_{s}\left(a, t_{s}\right)=0$ and that $\sup _{a \in A} g_{s}(a, t)=H\left(x, P_{x, s, U}(t)\right)$.

2. $\exists k \in[1 . . n]$ such that $f_{k}\left(x, a_{s}\right)=0$ (in other words $a_{s}$ is on the boundary of $A s$ ): In this case, $t_{s}=\min t_{s}^{i}$, where for each $i \in[1 . . n], t_{s}^{i}$ is the solution of the equation in $t$ : $\sup _{a \in A_{s}^{i+}} g_{s}(a, t)=0$ where $A_{s}^{i+}=A_{s}^{i} \cap A_{s}$ and $A_{s}^{i}=\left\{a \in A \mid f_{i}(x, a)=0\right\}$. 
In practice, this suggests the following protocol: First, we compute the roots $\overline{t_{s}}$ of $H\left(x, P_{x, s, U}(t)\right)=0$. Then, we test if the associated optimal control $\overline{a_{s}}$ is in $A_{s}$ (it is in $A$ and not necessarily in $A_{s}$ ). To do that, we just have to estimate the associated optimal dynamics

$$
f\left(x, \overline{a_{s}}\right)=-\nabla H\left(x, P_{x, s, U}\left(\overline{t_{s}}\right)\right)
$$

and to verify that $\forall k, \operatorname{sign}\left(f_{k}\left(x, \overline{a_{s}}\right)\right)=s_{k}$. We should note that, thanks to equation (24), the estimation of the associated optimal dynamics does not require the preliminary knowledge or computation of the associated optimal control $\overline{a_{s}}$. In particular, the test "Is $\overline{a_{s}}$ in $A_{s}$ ?" does not require the knowledge of the optimal control $\overline{a_{s}}$. Moreover, let us stress that this step directly provides the associated optimal dynamics. If all the signs are correct, we have found our solution and we stop here $\left(t_{s}=\overline{t_{s}}\right.$ ). Otherwise, we have to compute the solutions $t_{s}^{i}$ (defined above in item 2). To achieve this goal, we can rewrite

$$
H_{s}^{i}(x, p) \stackrel{\text { def }}{=} \sup _{a \in A_{s}^{i}}\{-f(x, a) \cdot p-l(x, a)\}=0
$$

in an explicit form (for example, by using differential calculus and the Legendre Transform as in Prados and Soatto (2005)) and deal with $H_{s}^{i}$ in the same way we have dealt with $H$. More precisely, we have to solve the equation

$$
\sup _{a \in A_{s}^{i} \mid \forall k, s_{k}(x, a)=s_{k}} g_{s}(a, t)=0 ;
$$

in the previous case, we had to solve

$$
\sup _{a \in A \mid \forall k, s_{k}(x, a)=s_{k}} g_{s}(a, t)=0 .
$$

$H_{s}^{i}$ plays the same role in equation (26) as $H$ in equation (27).

This process necessarily terminates because, at each stage, we reduce the dimension of the set of admissible controls. In our problem, the initial dimension of the set of admissible controls is 3: At the beginning the set of admissible controls is a tetrahedron; if the tests of the signs successively fail, the set of admissible controls we have to deal with becomes a triangle, and then a segment. Finally, we note that the rigorous management of all the sub-cases is fundamental in order to keep the scheme consistent. Also, generally we have $H_{s}^{i}(x, p) \neq H\left(x,\left(p_{1}, \ldots, p_{i-1}, 0, p_{i+1}, . ., p_{n}\right)\right)$. In particular, even if $H_{s}^{i}(x, p)=$ 
$H_{s}^{i}\left(x,\left(p_{1}, \ldots, p_{i-1}, 0, p_{i+1}, . ., p_{n}\right)\right)$, we have $H_{s}^{i}(x, p) \leq H\left(x,\left(p_{1}, \ldots, p_{i-1}, 0, p_{i+1}, . ., p_{n}\right)\right)$ and generally this inequality is strict. For a better understanding of the actual implementation of the method, we invite the interested reader to take a look at the $\mathrm{C}++$ code of this algorithm. It is freely distributed on the INRIA Gforge website.

\subsubsection{Details for the 3D anisotropic Eikonal equation}

Here we detail the successive "sided" Hamiltonians necessary for the implementation of the method described in the previous section (computation of the $t_{s}$ ) for the $3 \mathrm{D}$ anisotropic Eikonal equation. In this case, the control and the subset $A_{s}$ of the set $A$ are also of dimension 3. We have to separate the cases where the optimal dynamics is inside the volume $A_{s}$, on one of its faces or on one of its edges.

Let us remind that for any Hamiltonian $H(x, p)$, we call the Legendre Transform the function $H^{*}$ defined by

$$
H^{*}(x, a)=\sup _{p \in \operatorname{Dom}(H(x, .))}\{p \cdot a-H(x, p) \leq+\infty\}
$$

see for example Ekeland and Temam (1974), Prados (2004). Let us consider here

$$
H(x, p)=\left|A_{x} p\right|_{E}^{2}-1
$$

- In the case where the optimal dynamics is on the face of a volume $A_{s}$ defined by $a_{i}=0$, we have to use

$$
\begin{gathered}
H_{i}(x, p) \stackrel{\text { def }}{=} \sup _{a \in \mathbb{R}^{3}}\left\{a \cdot p-H^{*}(x, a)\right\}=\tilde{p}^{i T}\left(\llbracket D_{x}^{-1} \rrbracket^{i}\right)^{-1} \tilde{p}^{i}-1, \\
a_{i}=0
\end{gathered}
$$

- In the case where the optimal dynamics is on the edge of a volume $A_{s}$ defined by $a_{i}=0$ and $a_{j}=0$, we have to use

$$
\begin{gathered}
H_{i j}(x, p) \stackrel{\text { def }}{=} \sup _{a \in \mathbb{R}^{3}}\left\{a \cdot p-H^{*}(x, a)\right\}=\frac{p_{k}^{2}}{\left(D_{x}^{-1}\right)_{k, k}}-1, \\
a_{i}=0, a_{j}=0, i \neq j
\end{gathered}
$$


where $\tilde{p}^{i}=\left(p_{1}, \ldots, p_{i-1}, p_{i+1}, \ldots, p_{n}\right)$, and $\llbracket D_{x}^{-1} \rrbracket^{i}$ is the matrix $D_{x}^{-1}$ without the $i^{\text {th }}$ row and $i^{\text {th }}$ column.

In practice our method boils down to solving basic second order equations and testing some signs.

\subsection{Connectivity Measures Computation}

In this section we detail how to compute the connectivity measure $\mathcal{R}(\tilde{x})$ at the step 4 of our global algorithm. At this stage, we already know the optimal dynamics $f_{\tilde{x}}^{*}$, the optimal simplex $\left(\tilde{x}, x_{1}, x_{2}, x_{3}\right)$ (we denote $x_{i}=\tilde{x}+s_{i}(\tilde{x}) h_{i} e_{i}$ where $s_{i}(\tilde{x})$ is the sign of the $i^{\text {th }}$ component of $f_{\tilde{x}}^{*}$ and $h_{1} \times h_{2} \times h_{3}$ is the size of the voxels) and the values $\mathcal{R}\left(x_{i}\right)$ for $i=1 . .3$.

Let $y$ be the intersection of the optimal trajectory with the front. By assuming that the trajectory is locally affine, we have: $y=\tilde{x}+\tau f_{\tilde{x}}^{*}$ where $\tau$ is the time for the trajectory to reach the front, see figure 1(a). As in Prados et al. (2002), we can prove that

$$
\tau=1 / \sum_{i=1 . .3} q_{i}
$$

where $q_{i}$ is the absolute value of the $i^{t h}$ component of $f_{\tilde{x}}^{*}$ divided by $h_{i}$. By assuming that $\mathcal{R}$ is locally affine, we have (Prados et al. (2002))

$$
\mathcal{R}(y)=\sum_{i=1}^{3} \tau q_{i} \mathcal{R}\left(x_{i}\right) .
$$

Thus by noting that

$$
\mathcal{R}(\tilde{x})=\mathcal{R}(y)+\int_{0}^{\tau} \mathcal{C}\left(\xi_{\tilde{x}}^{*}(t)\right) d t
$$

we obtain

$$
\mathcal{R}(\tilde{x}) \simeq \sum_{i=1}^{3} \tau q_{i} \mathcal{R}\left(x_{i}\right)+\tau \mathcal{C}(\tilde{x})
$$

Remark 5. 1) The approximation of $\mathcal{S}(\tilde{x})$ required for the computation of the standard deviation $\sigma(\tilde{x})$ is obtained exactly in the same way. We just have to replace $\mathcal{C}$ by $\mathcal{C}^{2}$ in equation (28). 
2) This scheme can also be obtained by discretizing the equation $\left\langle\nabla_{E} \mathcal{R}(x), f_{x}^{*}\right\rangle_{E}=\mathcal{C}(x)$ (obtained by evaluating $\lim _{\varepsilon \rightarrow 0} \frac{\mathcal{R}\left(x+\varepsilon f_{x}^{*}\right)-\mathcal{R}(x)}{\varepsilon}$ ) and by slightly modifying the scheme proposed by Adalsteinsson and Sethian (1999) for solving a similar equation.

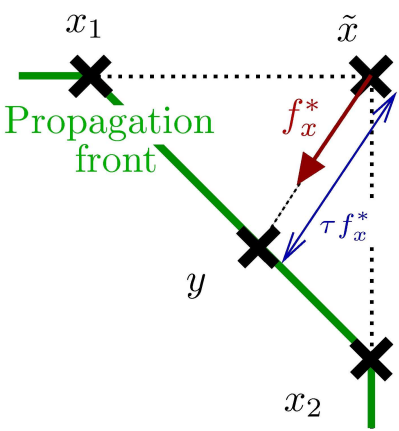

(a)

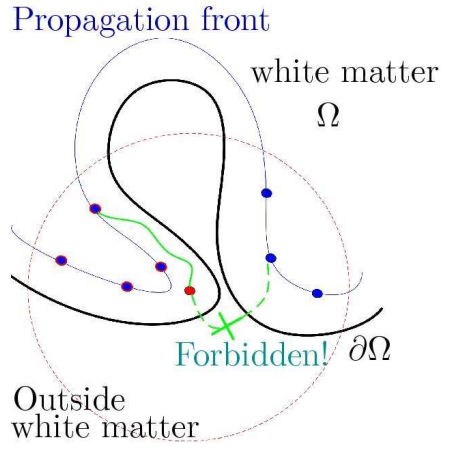

(b)

Figure 1: (a) Approximation of the geodesic, (b) Topological problem in a convoluted area of the white matter.

\section{Experimental Results}

In this section, we first present some results illustrating the computation of the distance function and geodesics by the method proposed in section 3. We then emphasize its possible limitations. Finally, we show that the algorithm introduced in the previous section can efficiently solve those issues.

\subsection{The Level Set Perspective}

We will consider synthetic and real DTI datasets to illustrate and quantify the quality of the estimated distance functions with upwind or fifth order WENO finite differences schemes. Our criterion will be the a posteriori evaluated map $|\operatorname{grad} u|$ which must be equal to 1 everywhere except at the origin $x_{0}$ since the distance function is not differentiable at this location. Figures 2 and 3 present a synthetic and a real DTI dataset and the associated 

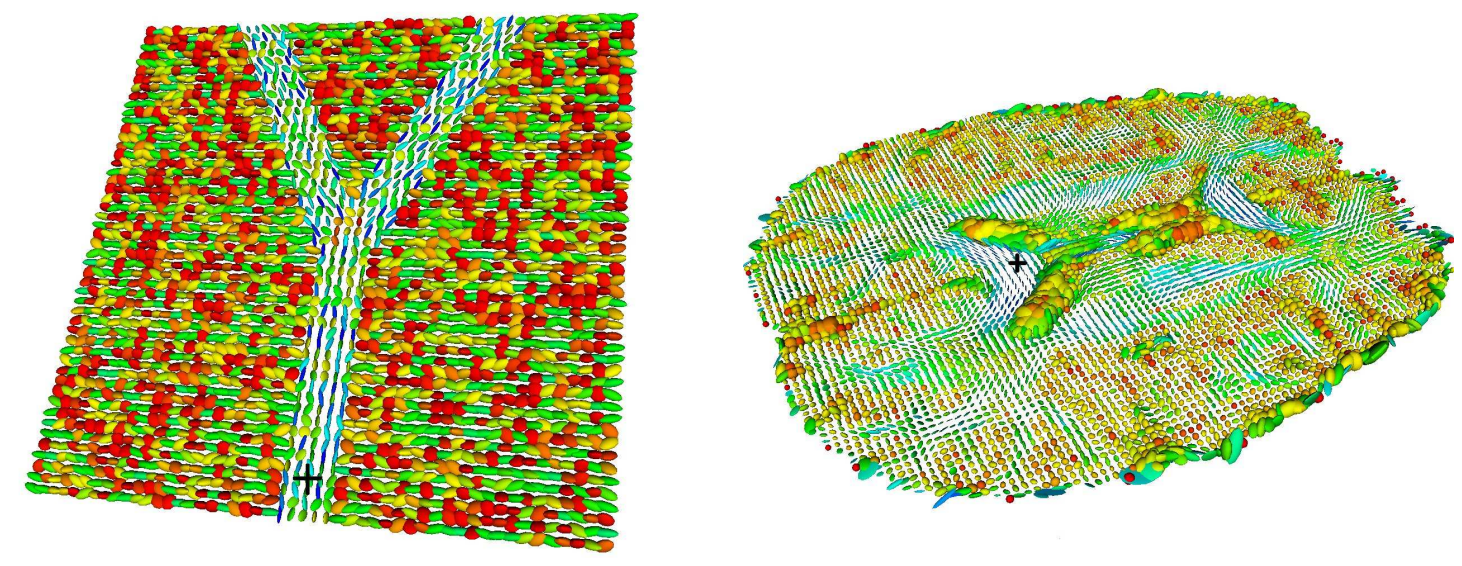

Figure 2: Synthetic (left) and human brain (right) DTI datasets.
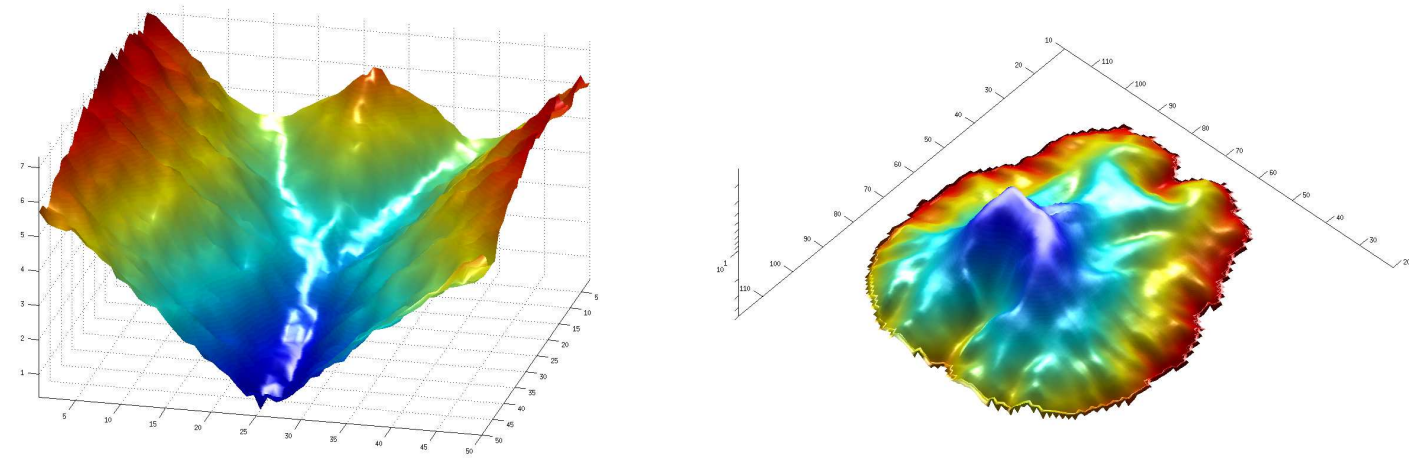

Figure 3: Axial slices of distance functions computed for the synthetic (left) and real (right) datasets of figure 2 (Color map: blue is small and red is large).

distance functions. The synthetic tensor field is composed by Y pattern with tensors aligned along the main orientations of this pattern. The background is made of tensors following the direction orthogonal to the main branch of the Y. Gaussian noise was added to the initial piecewise constant dataset in the tensor domain. We used the algorithm proposed in section 5.4.1 of Lenglet (2006). The origin $x_{0}$ was chosen to be at the lower part of the main branch of the Y (black cross). The resulting distance function is shown in figure 3 (left) where we represent one axial slice of the volume as an elevation surface to emphasize the variations of the function. We can notice that the distance stays small within the Y pattern and rapidly increases as it reaches the background. 
The human brain DTI dataset was provided by ${ }^{1}$ J.F. Mangin and J.B Poline. We focused on the posterior part of the corpus callosum, known as the genu. The origin $x_{0}$ of the distance was chosen in the middle of the genu (black cross) and the resulting distance function is presented in figure 3 (right) as an elevation surface (inverted for the sake of clarity). As expected, from neuroanatomical knowledge, the distance functions stays small along the commissural radiations towards the occipital areas of the brain and increases otherwise. By initiating the back-propagation procedure of section 3.2 in the visual area for instance (see chapter 11 of Lenglet (2006) for details on that point), or more generally in regions of the occipital part of the brain, we could recover the geodesics of figure 4, which agree with the overall well-known shape of neural fibers in these regions. Similarly to stochastic tractography techniques, where several thousands of streamlines are typically started from a single voxel and can reach almost any other location of the brain, our method yields connections between every single pair of points. In figure 4 , all the curves pass through the origin, in the splenium of the corpus callosum. We believe this is a good way to exhaustively test all possible connections and acknowledge that many of them, in fact, do not represent true fiber pathways and must be discarded. This is precisely the goal of the connectivity measures described in section 4.5. However, given the spatial and angular resolution of DTI, it is well-known that fiber crossing or fanning is rather likely at each voxel. One of the strengths of our technique is its flexibility and ability to capture such configurations to a certain extent.

Finally, we compared the accuracy of the distance computation that can be achieved with upwind or WENO schemes. We used the previous human brain DTI dataset but resorted to a more complex synthetic example. This dataset is made of three orthogonal and intersecting cylinders oriented along the $x, y$ and $z$ axis of the volume. Tensors inside each cylinder are aligned with the long axis of the cylinder and the background is made of small isotropic tensors. This results in perfectly isotropic tensors at the intersection of the three cylinders, surrounded by planar tensors in the area where only two cylinders cross

\footnotetext{
${ }^{1}$ Neurospin-CEA Saclay, France
} 


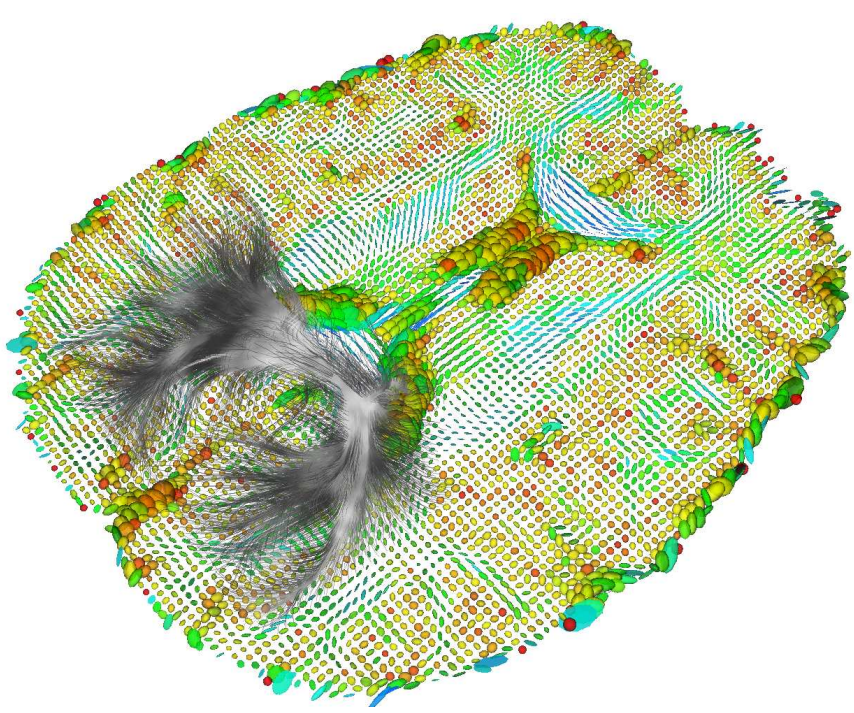

Figure 5: Cylinder dataset ten-

Figure 4: Geodesic paths of figure 3 (right).
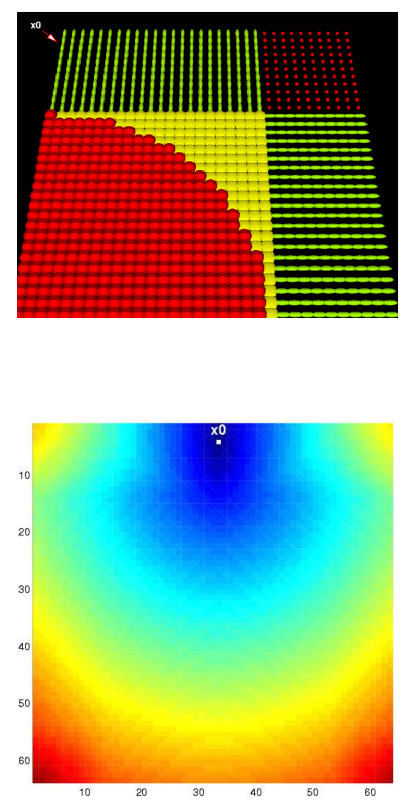

sor field (top) and distance function (bottom).

each others (see example of axial slice in top row of figure 5). The origin $x_{0}$ was chosen at one of the extremity of one cylinder and an axial slice of the computed distance function is presented in the bottom row of figure 5. Our numerical scheme 9 behaves fairly well on the two datasets. Table 1 shows the mean value, standard deviation and extrema of the norm of the intrinsic gradient of the distance function. By definition of the Eikonal equation, this should be equal to 1 everywhere in the volume, so these quantities give us a more precise idea of the stability and accuracy of each scheme. A sensible improvement is noticeable when using fifth order WENO schemes.

\subsection{Challenging Computational Issues}

The nature of the problem we are trying to solve raises two major computational difficulties which, to our knowledge, are not very well dealt with in the literature. They may also be an issue, in certain circumstances, for the dynamic formulation of the anisotropic Eikonal equation. 


\begin{tabular}{|c|c|c|c|c|}
\hline DataSet & Scheme & Mean & Std. Dev & Maximum \\
\hline Synthetic & Upwind & 0.9854 & 0.123657 & 4.50625 \\
\hline Synthetic & WENO & 0.977078 & 0.116855 & 2.0871 \\
\hline Real DTI & Upwind & 0.994332 & 0.116326 & 4.80079 \\
\hline Real DTI & WENO & 0.973351 & 0.110364 & 3.72567 \\
\hline
\end{tabular}

Table 1: Statistics on $|\operatorname{grad} u|$ for synthetic and real DTI datasets.

\subsubsection{Handling the white matter convoluted geometry}

First of all, as presented in figure 6 and detailed in figure 1(b), solving the anisotropic Eikonal equation within a convoluted domain such as the brain white matter is necessary and complicated. Indeed, the connections we are looking for are defined between cortical areas or between cortical areas and subcortical gray matter structures. In other words, we are essentially interested in pathways linking together parts of the domain boundary.

In figure 6 , the geodesic distance to the blue cross in image (b) (i.e. $x_{0}$ ) was computed, for the DTI data presented in image (a) and within the mask outlined in red in image (b). Its isovalues (in the range $[0,1500]$ ) are depicted by the yellow lines in images (c) and (d). With the level set implementation, the front diffuses through the cerebrospinal fluid and directly connects the right hemisphere. This is anatomically incorrect since the fibers starting from the blue cross (located in the V1 visual area) go through the corpus callosum (CC) to reach the other hemisphere. With the stationary formulation, we can correctly estimate the distance since, by definition, the Fast Marching implementation ignores all the locations outside the mask.

This kind of difficulty is also encountered with the Ordered Upwind Method (OUM) recently proposed by Sethian and Vladimirsky (2003). The OUM is a numerical method of type FMM which uses enlarged neighborhoods. The more anisotropic the tensor, the larger the neighborhood. In addition to increasing the computation time, Sethian and Vladimirsky's method explicitly authorizes this type of topological error by allowing the trajectories to step outside the mask and to directly connect any nearby voxel located on the front. Figure 1(b) illustrates 
this potential problem. The scheme presented in section 5 only uses nearest neighbors (six nearest neighbors in 3D). It is thus not prone to this problem and always respects the topology of the mask.

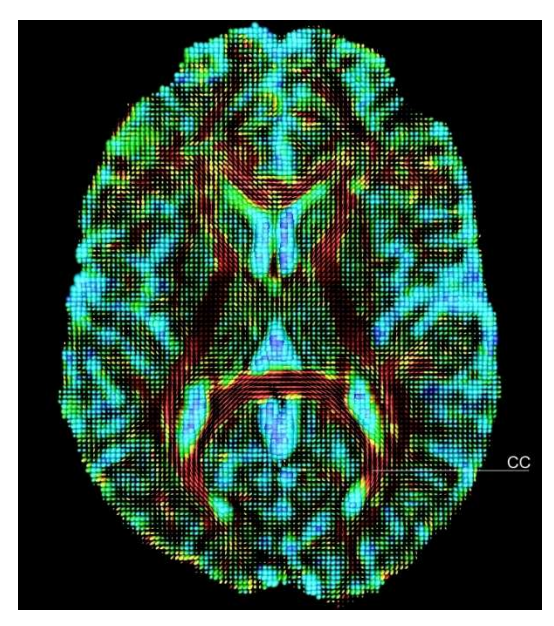

(a) DTI axial slice (Anisotropy color code: blue=low/red=high)

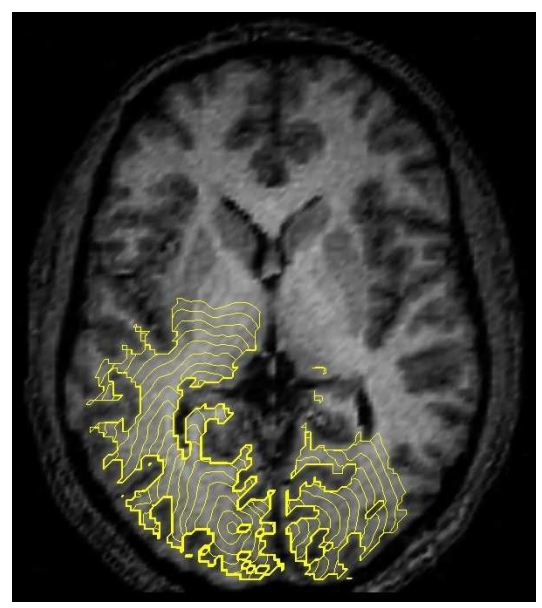

(c) Level set algorithm

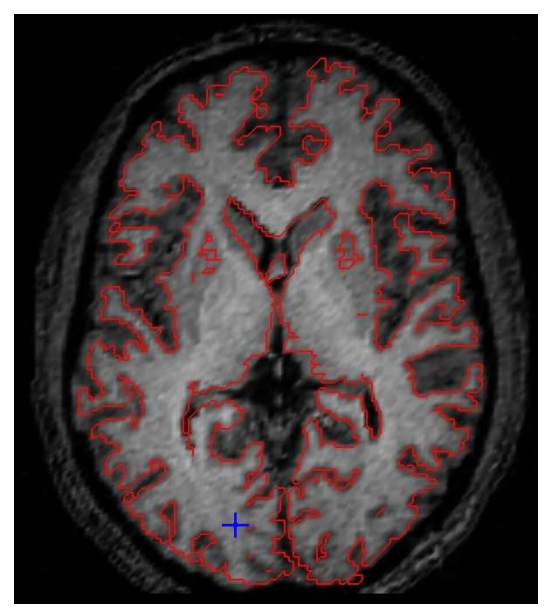

(b) White matter segmentation

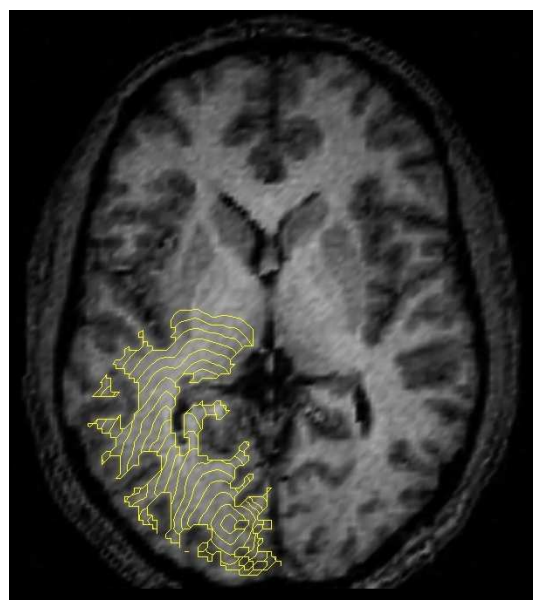

(d) Fast Marching algorithm

Figure 6: Topological inconsistency in the occipital cortex.

\subsubsection{Robust estimation of the optimal dynamics}

The second issue is related to the robustness of the optimal dynamics (i.e. the geodesics tangent vectors) computation. Indeed, at the exception of Pichon et al. (2005), Melonakos et al. (2007) who estimate the dynamics at the same time as the distance function, all the existing methods need to explicitly compute the derivatives of the distance function. This is well- 
known to be sensitive to noise, especially on the boundaries where the discretization of the differential needs to be adapted. We present, in figure 7, a comparison of the vector fields obtained by the method proposed in section 3 (top row) and by the approach of section 5 (bottom row) on a 3D synthetic DTI dataset (see image (a)). The origin of the distance function is located at the center of region B (see image (b)).

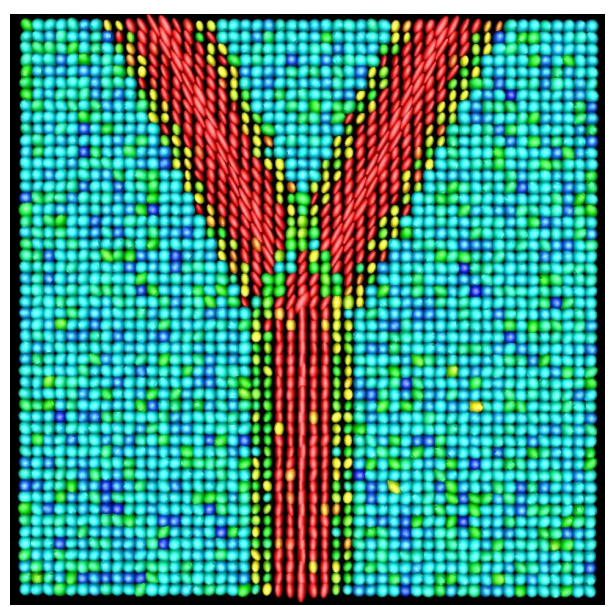

(a) DTI axial slice (Anisotropy color code: blue=low/red=high)

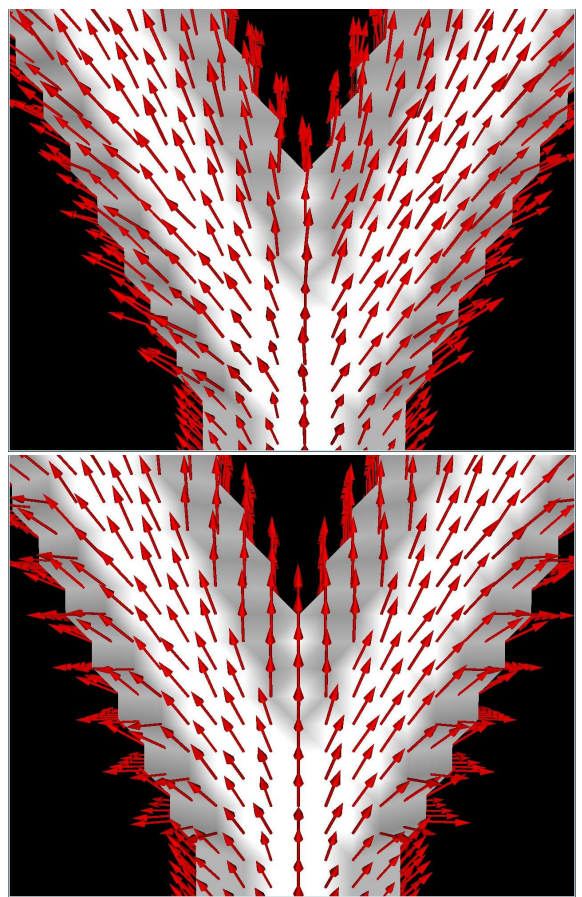

(c) Region A

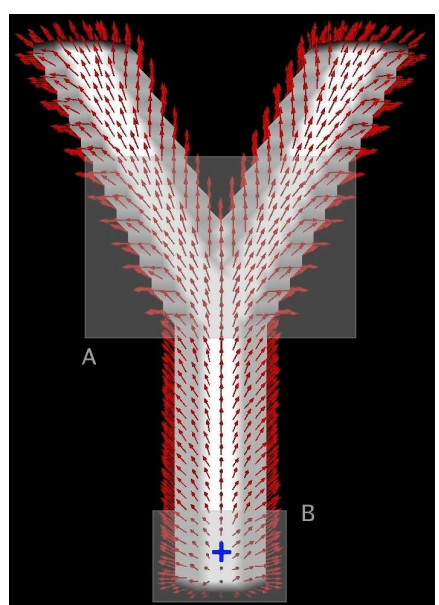

(b) Optimal dynamics
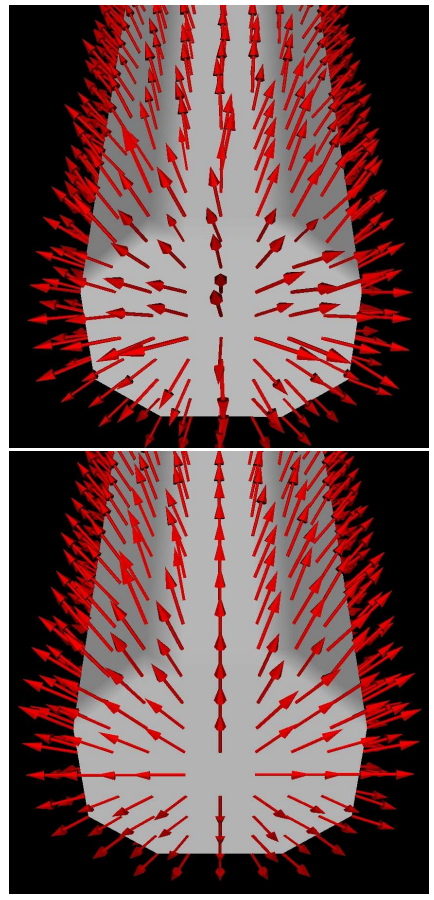

(d) Region B

Figure 7: Optimal dynamics estimation by differentiation of the distance (top) and by the control theory formulation (bottom). 


\subsection{The Fast Marching Perspective}

In the following, we illustrate our Fast Marching method by computing the quantities $\mu$ and $\sigma$, introduced in section 4.5, as well as the geodesics associated with the highest connectivity. This is done on the synthetic tensor field of figure 7 as well as in the splenium (posterior part) of the corpus callosum for the real dataset of figure 6. Diffusion weighted images were acquired at 3 Tesla at the Centre IRMf de Marseille, France, using a quadrature bird-cage head coil. We used 12 diffusion gradient directions and a $b$ factor of $1000 \mathrm{~s} / \mathrm{mm}^{2}$. Acquisitions were repeated 8 times for each direction in order to ensure a good signal-to-noise ratio. Voxel size was $2 \times 2 \times 2 \mathrm{~mm}^{3}$ and diffusion tensors were estimated with the robust gradient descent algorithm proposed in Lenglet et al. (2006), which naturally enforces positivity by working on the manifold of symmetric positive-definite matrices. An axial slice of the resulting DT image is presented in figure $6(\mathrm{a})$.

\subsubsection{Computational efficiency}

PDE methods for brain connectivity mapping such as O’Donnell et al. (2002), Parker et al. (2002b), Lenglet et al. (2004), Jackowski et al. (2005), Campbell et al. (2005), Fletcher et al. (2007) have the great advantage to yield connectivity information for a point of interest $x_{0}$ to the rest of the brain by exploiting the full information of the diffusion tensor. They are however in general quite time consuming and must be iteratively applied to all the voxels of functional regions of interest, which can contain hundreds or thousands of points. By comparison with the methods presented in Jackowski et al. (2005) and Lenglet et al. (2004), our Fast Marching algorithm achieves a dramatic improvement in computational speed. For the geodesic distance computation, Jackowski et al. reported a convergence time of about 7 minutes for their iterative sweeping method for a $128 \times 128 \times 40$ DTI dataset on a $1.7 \mathrm{GHz}$ Intel Pentium Xeon with 1.5 Gb of RAM. Our level set formulation required about 20 minutes for a $128 \times 128 \times 58$ DTI dataset on a $1.7 \mathrm{GHz}$ Intel Pentium M with $1 \mathrm{~Gb}$ of RAM.

The computation of the geodesics, together with the evaluation of the statistics of $\mathcal{C}(x)$, 
is itself a time-consuming task since for each curve, we need to explicitly propagate through the tangent vectors field using, for instance, a $4^{\text {th }}$ order Runge-Kutta integration scheme. In Jackowski et al. (2005), no time is given for the computation of the 14,952 fibers of interest. However, on our data and for 135,029 voxels inside the white matter mask, it took approximately 30 minutes on the same computer than the one used for the distance computation. All these computations (distance, vector field and connectivity measures) take about 7 seconds with our stationary/control theory formulation.

\subsubsection{Performance of the connectivity measure}

We now demonstrate how the statistics of the quantity $\mathcal{C}(x)$ can be used to evaluate the degree of connectivity of pairs of voxels. First of all, we use the synthetic dataset of figure 7. The
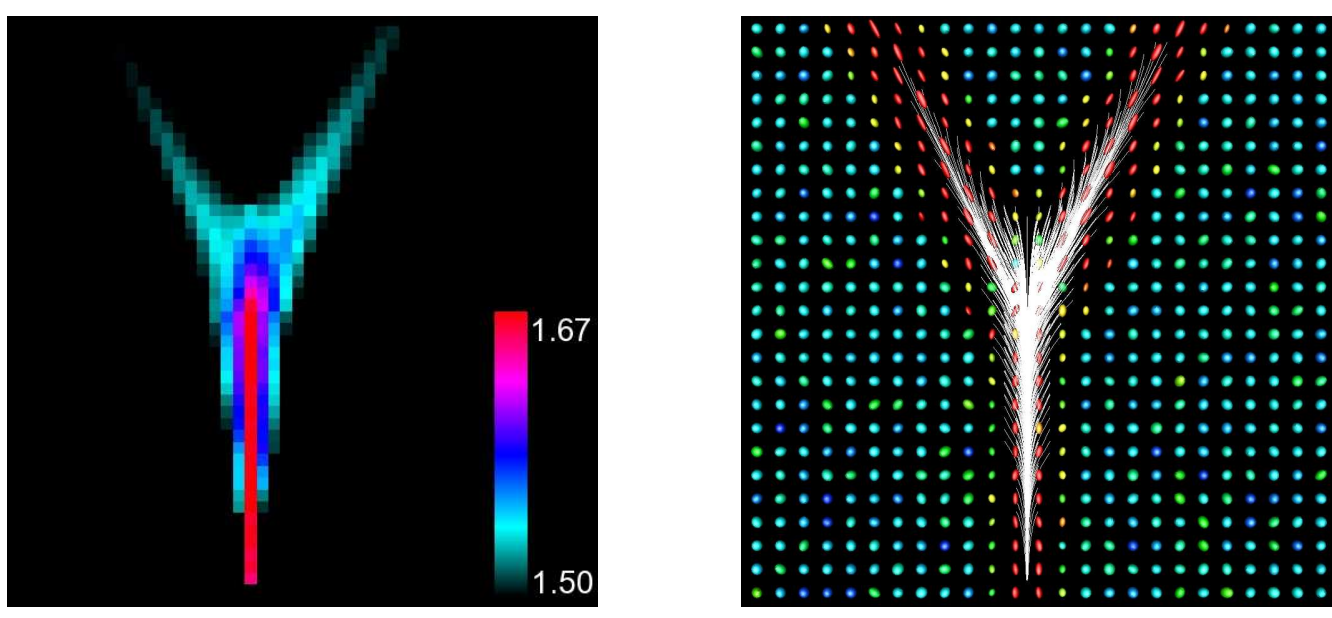

Figure 8: Synthetic dataset: Axial slice of the map $\mu$ (left), Most likely connections (right).

point of interest $x_{0}$ is again located at the center of region B (see image (b)). Figure 8 (left) presents an axial slice of the thresholded map $\mu$ which is consistent with the DT image since we can see that $\mu$ is higher along the centerline of the $Y$ shape where the tensors are more anisotropic. Moreover, the right branch is clearly more connected to the origin. This is due to the asymmetry imposed by the tensor field in the diverging region (see figure 7 (a)). In figure 8 (right), we show the geodesics computed from the 873 voxels with values of $\mu$ in the range $[1.5,1.67]$, i.e. the top $10 \%$ most likely connected voxels. Finally, we consider the real dataset of figure 9. The origin is located in the middle of the splenium of the corpus callosum. 

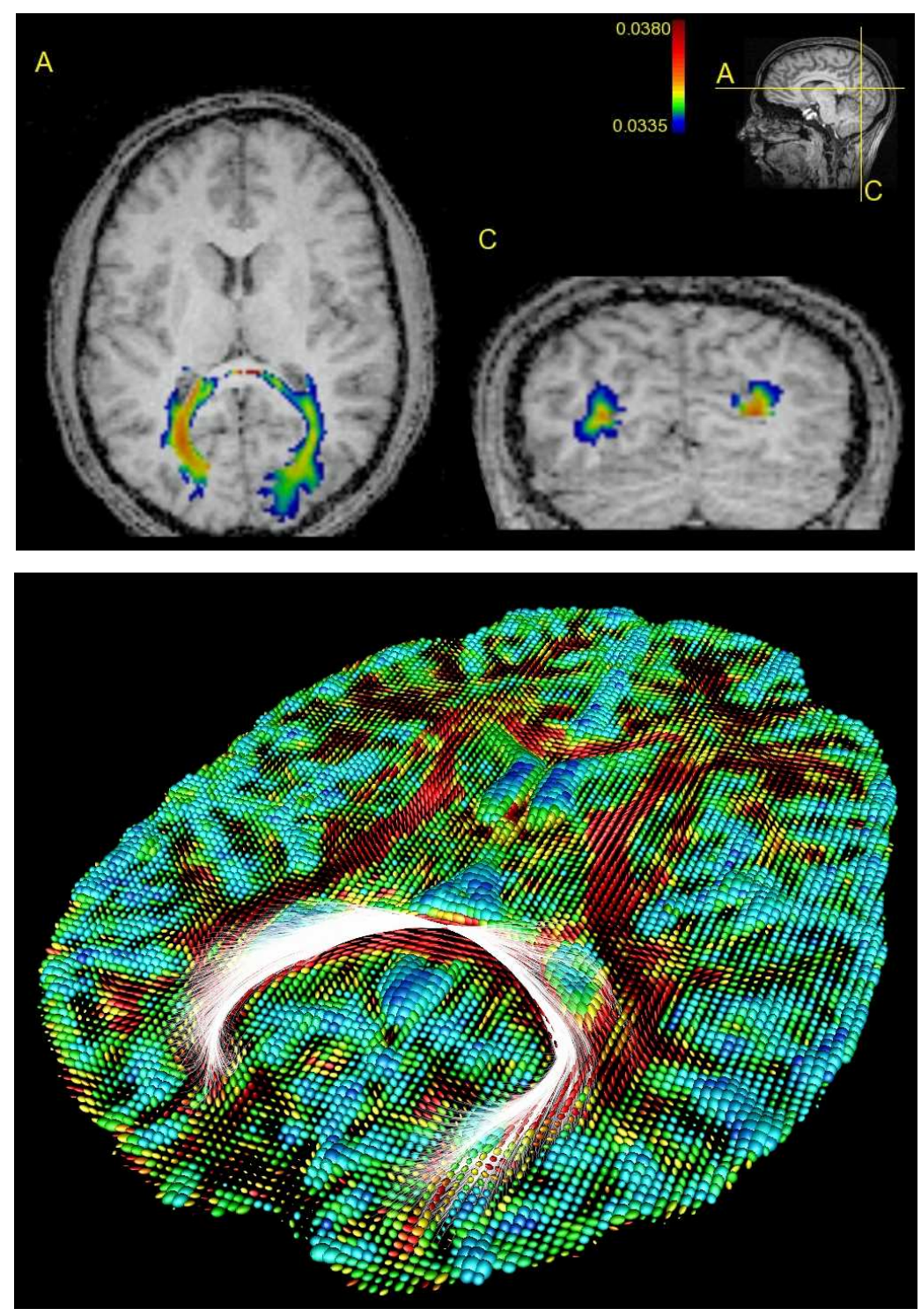

Figure 9: Real dataset: Axial and coronal slices of the map $\mu$ (top), Most likely connections (bottom).

A first threshold is applied on the map $\sigma$ in order to keep only coherent fibers. This yields a binary mask (threshold value: 0.0056) which is applied to the map $\mu$. As previously, we then threshold this map to preserve only the top $10 \%$ most likely connected voxels, with values of $\mu$ in the range $[0.0335,0.0380]$. This yields 2561 fibers that are largely consistent with neuroanatomical knowledge. As already acknowledged in section 6.1, many of the connections initially computed between the corpus callosum and the rest of the brain obviously do not represent true neural pathways. After thresholding of the $\sigma$ and $\mu$ connectivity maps, the 
result presented in figure 9 is however reasonably representative of the actual fiber bundle.

\section{Conclusion}

We have proposed a novel global approach to white matter connectivity mapping. It relies on the fact that probing and measuring a diffusion process on a manifold $\mathcal{M}$ provides enough information to infer the geometry of $\mathcal{M}$ and compute its geodesics, corresponding to diffusion pathways. We introduced a dynamic formulation of the anisotropic Eikonal equation and a method to approximate neural fibers. Exploiting both an optimal control and a Riemannian interpretation, we achieved a number of improvements over existing methods. We proposed a fast algorithm that reduces CPU time by 2 or 3 orders of magnitude by comparison with existing works. We have introduced a general local connectivity measure and experimentally demonstrated its relevance on real data sets. Our algorithm is numerically stable and efficient, since it simultaneously computes the distance function, the optimal dynamics and the statistics of our local connectivity measure. Finally, we showed that our method overcomes some numerical limitations which cause existing algorithms to fail in highly convoluted regions. The C++ implementation of our GCM algorithm is freely distributed on the INRIA Gforge website.

\section{A Proof of proposition 5.3.1:}

For the convenience of the reader, let us note that for all $a \in A_{s}$,

$$
-f(x, a) \cdot P_{x, s, U}(t)=t\left(\sum_{i=1 . . n} \frac{\left|f_{i}(x, a)\right|}{h_{i}}\right)-\sum_{i=1 . . n} \frac{\left|f_{i}(x, a)\right|}{h_{i}} u\left(x+s_{i} h_{i} e_{i}\right) .
$$

Also, we can rewrite $g_{s}(a, t)$ as

$$
g_{s}(a, t)=t\left(\sum_{i=1 . . n} \frac{\left|f_{i}(x, a)\right|}{h_{i}}\right)-\sum_{i=1 . . n} \frac{\left|f_{i}(x, a)\right|}{h_{i}} u\left(x+s_{i} h_{i} e_{i}\right)-l(x, a) .
$$

We first prove the existence: Let us assume that there exists $t_{+}$and $t_{-}$such that $\sup _{a \in A_{s}} g_{s}\left(a, t_{+}\right)>0$ and $\sup _{a \in A_{s}} g_{s}\left(a, t_{-}\right)<0$; the existence then follows from the intermediate value theorem and the continuity of the function $t \mapsto \sup _{a \in A_{s}} g_{s}(a, t)$ (guaranteed by the 
concavity and the strong regularity of $g_{s}(a, t)$ with respects to $t$ and $a$ ).

We now prove the existence of $t_{-}$: By contradiction, let us assume that for all $t \in \mathbb{R}$

$$
\sup _{a \in A_{s}} g_{s}(a, t) \geq 0 .
$$

- First, let us state that, when $|f(x, a)|$ is small, $-l(x, a)$ is strictly negative. For $f$ and $l$ defined as in section 4.3 , we can easily verify that, for $a$ such that $f(x, a)=0$, we have $-l(x, a)<0$. In other words, we have $-l\left(x,[f(x, .)]^{-1}(0)\right)<0$, where $[f(x, .)]^{-1}$ is the inverse function of $f(x,$.$) . By continuity of the function b \mapsto-l\left(x,[f(x, .)]^{-1}(b)\right)$, it follows that for any $\delta$ s.t. $-l\left(x,[f(x, .)]^{-1}(0)\right)<\delta<0$ there exists $\varepsilon>0$ such that if $|f(x, a)| \leq \varepsilon$ then $-l(x, a)<\delta<0$.

Also, similarly we can get: For all $a$ such that $\sum_{i=1 . . n} \frac{\left|f_{i}(x, a)\right|}{h_{i}} \leq \varepsilon$, we have $-l(x, a)<\delta<$ 0.

- Then we prove that if $t$ is small then $|f(x, a)|$ is also small. Let us consider any $t$ such that $\sum_{i=1 . . n} \frac{\left|f_{i}\left(x, a_{t}\right)\right|}{h_{i}}>\varepsilon$, where $a_{t}=\arg \sup _{a \in A_{s}} g_{s}(a, t)$. By the assumption (29), we have

$$
\begin{aligned}
t \geq\left(\sum_{i=1 . . n} \frac{\left|f_{i}\left(x, a_{t}\right)\right|}{h_{i}}\right)^{-1} \sum_{i=1 . . n} \frac{\left|f_{i}\left(x, a_{t}\right)\right|}{h_{i}} u\left(x+s_{i} h_{i} e_{i}\right) & \\
& -\left(\sum_{i=1 . . n} \frac{\left|f_{i}\left(x, a_{t}\right)\right|}{h_{i}}\right)^{-1} l\left(x, a_{t}\right) .
\end{aligned}
$$

The first part of the above equation is a barycentric combination of the $u\left(x+s_{i} h_{i} e_{i}\right)$. Thus we can easily obtain

$$
t \geq \min _{i} u\left(x+s_{i} h_{i} e_{i}\right)-B \varepsilon^{-1},
$$

where $B$ is an upper bound of $|l(x,)$.$| .$

- We now easily prove that if $t$ is small then $g_{s}\left(a_{t}, t\right)$ is strictly negative and then get the contradiction. Let us fix $t$ such that $t<\min _{i} u\left(x+s_{i} h_{i} e_{i}\right)-B \varepsilon^{-1}$. By the previous item we have $\sum_{i=1 . . n} \frac{\left|f_{i}\left(x, a_{t}\right)\right|}{h_{i}} \leq \varepsilon$ and so, according to the first item, $-l\left(x, a_{t}\right)<\delta<0$. Thus,

$$
g_{s}\left(a_{t}, t\right) \leq\left[t-\min _{i} u\left(x+s_{i} h_{i} e_{i}\right)\right]\left(\sum_{i=1 . . n} \frac{\left|f_{i}\left(x, a_{t}\right)\right|}{h_{i}}\right)-l\left(x, a_{t}\right),
$$


and

$$
\sup _{a \in A_{s}} g_{s}(a, t)=g_{s}\left(a_{t}, t\right) \leq-B \varepsilon^{-1}\left(\sum_{i=1 . . n} \frac{\left|f_{i}\left(x, a_{t}\right)\right|}{h_{i}}\right)-\delta \leq \delta<0
$$

which is a contradiction.

Next, we prove the existence of $t_{+}$: It is sufficient to find $a$ and $t$ such that $g_{s}(a, t)>0$. Also, for any $a$ such that $f(x, a) \neq 0$, when $t \rightarrow+\infty$, we have $-f(x, a) \cdot P_{x, s, U}(t) \rightarrow+\infty$ and so, $g_{s}(a, t) \rightarrow+\infty$. The conclusion follows.

Finally, we prove the uniqueness of $t_{s}$ : Let us assume by contradiction that there exist $t_{1}<t_{2}$, two solutions of (23). Let $a_{1}$ be the optimal control associated to $t_{1}$ (note that $f\left(x, a_{1}\right) \neq 0$ because we assume that $\left.-l\left(x,[f(x, .)]^{-1}(0)\right)<0\right)$. We have

$$
-f\left(x, a_{1}\right) \cdot P_{x, s, U}\left(t_{1}\right)-l\left(x, a_{1}\right)=0,
$$

thus

$$
-f\left(x, a_{1}\right) \cdot P_{x, s, U}\left(t_{2}\right)-l\left(x, a_{1}\right)>0,
$$

and, therefore

$$
\sup _{a \in A_{s}}-f(x, a) \cdot P_{x, s, U}\left(t_{2}\right)-l(x, a)>0
$$

which contradicts $t_{2}$ solution of (23).

\section{References}

D. Adalsteinsson and J.A. Sethian. The fast construction of extension velocities in level set methods. Journal of Computational Physics, 148(1):2-22, 1999.

A.L. Alexander, Hasan K.M., M. Lazar, J.S. Tsuruda, and D.L. Parker. Analysis of partial volume effects in diffusion-tensor MRI. Magnetic Resonance in Medicine, 45(5):770-780, 2001.

M. Bardi and I. Capuzzo-Dolcetta. Optimal control and viscosity solutions of HamiltonJacobi-Bellman equations. Birkhäuser Verlag, 1997.

P.J. Basser, J. Mattiello, and D. Le Bihan. Estimation of the effective self-diffusion tensor from the NMR spin echo. Journal of Magnetic Resonance, B(103):247-254, 1994. 
P.J. Basser, S. Pajevic, C. Pierpaoli, J. Duda, and A. Aldroubi. In vivo fiber tractography using DT-MRI data. Magnetic Resonance in Medicine, 44:625-632, 2000.

P.G. Batchelor, D.L.G. Hill, F. Calamante, and D. Atkinson. Study of connectivity in the brain using the full diffusion tensor from MRI. In Information Processing in Medical Imaging, pages 121-133, 2001.

T.E.J. Behrens, M.W. Woolrich, M. Jenkinson, H. Johansen-Berg, R.G. Nunes, S. Clare, P.M. Matthews, J.M. Brady, and S.M. Smith. Characterization and propagation of uncertainty in Diffusion-Weighted MR Imaging. Magnetic Resonance in Medicine, 50:1077-1088, 2003.

D. Le Bihan and E. Breton. Imagerie de diffusion in vivo par résonance magnétique nucléaire. Compte Rendu de l'Académie des Sciences, 301:1109-1112, 1985.

M. Bjornemo, A. Brun, R. Kikinis, and C.F. Westin. Regularized stochastic white matter tractography using diffusion tensor MRI. In International Conference on Medical Image Computing and Computer Assisted Intervention, pages 435-442, 2002.

J.S.W. Campbell, K. Siddiqi, V.V. Rymar, A. Sadikot, and B.G. Pike. Flow-based fiber tracking with diffusion tensor q-ball data: Validation and comparison to principal diffusion direction techniques. NeuroImage, 27(4):725-736, October 2005.

Y.G. Chen, Y. Giga, and S. Goto. Uniqueness and existence of viscosity solutions of generalized mean curvature flow equations. Journal on Differential Geometry, 33:749-786, 1991.

David L. Chopp. Computing minimal surfaces via level set curvature flow. Journal of Computational Physics, 106:77-91, 1993.

M.K. Chung, M. Lazar, A.L. Alexander, Y. Lu, and R. Davidson. Probabilistic connectivity measure in diffusion tensor imaging via anisotropic kernel smoothing. Technical Report 1081, Department of Statistics, University of Wisconsin-Madison, 2003.

L. Cohen. Minimal paths and fast marching methods for image analysis. In Mathematical Models in Computer Vision: The Handbook, chapter 7. Springer, 2005. 
R.W.R. Darling. Intrinsic location parameter of a diffusion process. Technical Report 493, UC Berkeley, Department of Statististics, 1998.

M. de Lara. Geometric and symmetry properties of a nondegenerate diffusion process. An. of Probability, 23(4):1557-1604, 1995.

M. Descoteaux, C. Lenglet, and R. Deriche. Diffusion tensor sharpening improves white matter tractography. In SPIE Medical Imaging, volume 6512, 2007.

M. P. Do Carmo. Riemannian Geometry. Birkhäuser Verlag, 1992.

P. Dupuis and J. Oliensis. An optimal control formulation and related numerical methods for a problem in shape reconstruction. The Annals of Applied Probability, 4(2):287-346, 1994.

A. Einstein. Investigations on the Theory of the Brownian Movement. New York: Dover, 1956. ISBN 048660304.

I. Ekeland and R. Temam. Analyse Convexe et Problèmes Variationnels. Etudes mathématiques. Dunod; Gauthier-Villars, Paris, Bruxelles, Montreal, 1974.

P.T. Fletcher, R. Tao, W.K. Jeong, and R.T. Whitaker. A volumetric approach to quantifying region-to-region white matter connectivity in diffusion tensor mri. In Information Processing in Medical Imaging, volume 4584, pages 346-358, July 2007.

O. Friman and C.F. Westin. Uncertainty in white matter fiber tractography. In International Conference on Medical Image Computing and Computer Assisted Intervention, pages 107114. Springer-verlag, 2005.

O. Friman, G. Farneback, and C.F. Westin. A Bayesian approach for stochastic white matter tractography. IEEE Transactions on Medical Imaging, 25:965-978, 2006.

N. Hageman, D. Shattuck, K. Narr, and A.W. Toga. A diffusion tensor imaging tractography method based on Navier-Stokes fluid mechanics. In IEEE International Symposium on Biomedical Imaging: From Nano to Macro, pages 798- 801, 2006. 
P. Hagmann, J.P. Thiran, L. Jonasson, P. Vandergheynst, S. Clarke, P. Maeder, and R. Meuli. DTI mapping of human brain connectivity: Statistical fiber tracking and virtual dissection. NeuroImage, 19:545-554, 2003.

A. Harten, B. Engquist, S. Osher, and S. Chakravarthy. Uniformly high-order accurate essentially non oscillatory schemes iii. Journal of Computational Physics, 71:231-303, 1987.

R. Iyer, R. Holsapple, and D. Doman. Optimal control problems on parallelizable Riemannian manifolds: theory and applications. ESAIM: Control, Optimisation and Calculus of Variations, 12:1-11, January 2006.

M. Jackowski, C.Y. Kao, M. Qiu, R.T. Constable, and L.H. Staib. White matter tractography by anisotropic wavefront evolution and diffusion tensors imaging. Medical Image Analysis, 9:427-440, 2005.

S. Jbabdi, P. Bellec, R. Toro, J. Daunizeau, M. Pélégrini-Isaac, and H. Benali. Accurate anisotropic fast marching for diffusion-based geodesic tractography. International Journal of Biomedical Imaging, 2008:320195, 2008.

W.K. Jeong and R. Whitaker. A fast eikonal equation solver for parallel systems. In SIAM Conference on Computational Science and Engineering, Costa Mesa, CA, February 2007.

D.K. Jones, A. Simmons, S.C.R. Williams, and M.A. Horsfield. Noninvasive assessment of axonal fiber connectivity in the human brain via diffusion tensor MRI. Magnetic Resonance in Medicine, 42:37-41, 1999.

N. Kang, J. Zhang, E.S. Carlson, and D. Gembris. White matter fiber tractography via anisotropic diffusion simulation in the human brain. IEEE Transactions on Medical Imaging, 24:1127-1137, 2005.

R. Kimmel and J. A. Sethian. Computing Geodesic Paths on Manifolds. Proceedings of the National Academy of Sciences, 95, 1998.

R. Kimmel, A. Amir, and A.F. Bruckstein. Finding shortest paths on surfaces using level 
set propagation. IEEE Transactions on Pattern Analysis and Machine Intelligence, 17(6): 635-640, June 1995.

M. Koch, V. Glauche, J. Finsterbusch, U. Nolte, J. Frahm, and C. Büchel. Estimation of anatomical connectivity from diffusion tensor data. In 7th Annual Meeting of the Organization for Human Brain Mapping, page 1974, 2001.

E. Konukoglu, M. Sermesant, O. Clatz, J.-M. Peyrat, H. Delingette, and N. Ayache. A recursive anisotropic fast marching approach to reaction diffusion equation: Application to tumor growth modeling. In IPMI, volume 4584 of Lecture Notes in Computer Science, pages 687-699. Springer, 2007.

M. Lazar and A. Alexander. Bootstrap white matter tractography (BOOT-TRAC). Neuroimage, 24:524-532, 2005.

M. Lazar and A.L. Alexander. White matter tractography using random vector (rave) perturbation. In 10th ISMRM Annual Meeting, page 539, 2002.

M. Lazar, D.M. Weinstein, J.S. Tsuruda, K.M. Hasan, K. Arfanakis, M.E. Meyerand, B. Badie, H.A. Rowley, V.Haughton, A. Field, and A.L. Alexander. White matter tractography using diffusion tensor deflection. Human Brain Mapping, 18(4):306-321, 2003.

S. Lehericy, C. Lenglet, J. Doyon, H. Benali, P.F. Van de Moortele, G. Sapiro, O. Faugeras, R. Deriche, and K. Ugurbil. Activation shifts from the premotor to the sensorimotor territory of the striatum during the course of motor sequence learning. In 11th Annual Meeting of the Organization for Human Brain Mapping, Toronto, June 2005.

C. Lenglet. Geometric and Variational Methods for Diffusion MRI Processing. PhD thesis, INRIA / University of Nice-Sophia Antipolis, December 2006.

C. Lenglet, R. Deriche, and O. Faugeras. Inferring white matter geometry from diffusion tensor MRI: Application to connectivity mapping. In European Conference on Computer Vision, pages 127-140, May 2004. 
C. Lenglet, M. Rousson, R. Deriche, and 0. Faugeras. Statistics on the manifold of multivariate normal distributions: Theory and application to diffusion tensor MRI processing. Journal of Mathematical Imaging and Vision, 25(3):423-444, October 2006.

R.J. LeVeque. Numerical Methods for Conservation Laws. Birkhäuser Verlag, Basel, 1992.

P.-L. Lions. Generalized Solutions of Hamilton-Jacobi Equations. Number 69 in Research Notes in Mathematics. Pitman Advanced Publishing Program, 1982.

X.D. Liu, S. Osher, and T. Chan. Weighted essentially non oscillatory schemes. J. Comput. Phys., 115:200-212, 1994.

J.-F. Mangin, C. Poupon, Y. Cointepas, D. Rivière, D. Papadopoulos-Orfanos, C. A. Clark, J. Régis, and D. Le Bihan. A framework based on spin glass models for the inference of anatomical connectivity from Diffusion-Weighted MR data. NMR in Biomedicine, 15:481$492,2002$.

C. Mantegazza and A.C. Mennucci. Hamilton-Jacobi equations and distance functions on Riemannian manifolds. App. Math. and Optim., 47(1):1-25, 2002.

T. McGraw and M. Nadar. Stochastic DT-MRI connectivity mapping on the gpu. IEEE Transactions on Visualization and Computer Graphics, 13(6):1504-1511, November-December 2007.

J. Melonakos, V. Mohan, M. Niethammer, K. Smith, M. Kubicki, and A. Tannenbaum. Finsler tractography for white matter connectivity analysis of the cingulum bundle. In International Conference on Medical Image Computing and Computer Assisted Intervention, pages 36-43, 2007.

K.D. Merboldt, W. Hanicke, and J. Frahm. Self-diffusion NMR imaging using stimulated echoes. J. Magn. Reson., 64:479-486, 1985.

S. Mori and P. van Zijl. Fiber tracking: Principles and strategies - a technical review. Nuclear Magnetic Resonance in Biomedicine, 15(7-8):468-480, 2003. 
S. Mori, B.J. Crain, V.P. Chacko, and P.C.M. Van Zijl. Three-dimensional tracking of axonal projections in the brain by Magnetic Resonance Imaging. Annals of Neurology, 45(2):265269, February 1999.

M.E. Moseley, Y. Cohen, J. Mintorovitch, J. Kucharczyk, J. Tsuruda, P. Weinstein, and D. Norman. Diffusion-weighted mr imaging of anisotropic water diffusion in cat central nervous system. Radiology, 176(2):439-445, 1990.

L. O’Donnell, S. Haker, and C.F. Westin. New approaches to estimation of white matter connectivity in Diffusion Tensor MRI: Elliptic PDEs and geodesics in a tensor-warped space. In International Conference on Medical Image Computing and Computer Assisted Intervention, 2002. 459-466.

S. Osher. A level set formulation for the solution of the Dirichlet problem for a HamiltonJacobi equations. SIAM J. Math. Anal., 24(5):1145-1152, 1993.

S. Osher and J.A. Sethian. Fronts propagating with curvature-dependent speed: Algorithms based on Hamilton-Jacobi formulations. Journal of Computational Physics, 79(1):12-49, 1988.

P.A. Osment, K.J. Packer, M.J. Taylor, J. J. Attard, T. A. Carpenter, L. D. Hall, S. J. Doran, and N. J. Herrod. NMR imaging of fluids in porous solids. Phil. Trans. Roy. Soc., 333:441-452, 1990.

G.J Parker, H.A. Haroon, and C.A Wheeler-Kingshott. A framework for a streamline-based probabilistic index of connectivity (PICo) using a structural interpretation of MRI diffusion measurements. Journal of Magnetic Resonance Imaging, 18(2):242-254, August 2003.

G.J.M. Parker and D.C Alexander. Probabilistic Monte Carlo based mapping of cerebral connections utilising whole-brain crossing fibre information. In Information Processing in Medical Imaging, pages 684-695, 2003.

G.J.M. Parker, K.E. Stephan, G.J. Barker, J.B. Rowe, D.G. MacManus, C.A.M WheelerKingshott, O. Ciccarelli, R.E. Passingham, R.L. Spinks, R.N. Lemon, and R. Turner. Initial 
demonstration of in vivo tracing of axonal projections in the macaque brain and comparison with the human brain using Diffusion Tensor Imaging and Fast Marching Tractography. NeuroImage, 15(4):797-809, April 2002a.

G.J.M. Parker, C.A.M. Wheeler-Kingshott, and G.J. Barker. Estimating distributed anatomical connectivity using Fast Marching Methods and Diffusion Tensor Imaging. Transactions in Medical Imaging, 21(5):505-512, 2002b.

M. Perrin, C. Poupon, Y. Cointepas, B. Rieul, N. Golestani, C. Pallier, D. Rivière, D. Le Bihan, and J.F. Mangin. Fiber tracking in q-ball fields using regularized particle trajectories. In Information Processing in Medical Imaging, pages 52-63, 2005.

E. Pichon, G. Sapiro, and A. Tannenbaum. Directions in Mathematical Systems Theory and Optimization, volume 286 of Lecture Notes in Control and Information Sciences, chapter Segmentation of Diffusion Tensor Imagery, pages 239-247. Springer-Verlag Heidelberg, 2003.

E. Pichon, C.F. Westin, and A. A. Tannenbaum. A hamilton-jacobi-bellman approach to high angular resolution diffusion tractography. In Proceedings of MICCAI'05, pages 180-187, 2005.

E. Prados. Application of the theory of the viscosity solutions to the Shape From Shading problem. PhD thesis, INRIA / University of Nice-Sophia Antipolis, 2004.

E. Prados and S. Soatto. Fast marching method for generic shape from shading. In IEEE Workshop on Variational and Level Set Methods, pages 320-331, 2005.

E. Prados, O. Faugeras, and E. Rouy. Shape from shading and viscosity solutions. Research Report 4638, INRIA Sophia Antipolis, November 2002.

E. Prados, C. Lenglet, J.P. Pons, N. Wotawa, E. Deriche, O. Faugeras, and S. Soatto. Control theory and fast marching methods for brain connectivity mapping. In IEEE Computer Society Conference on Computer Vision and Pattern Recognition, pages 1076-1083, New York, NY, June 2006a. 
E. Prados, C. Lenglet, J.P. Pons, N. Wotawa, R. Deriche, O. Faugeras, and S. Soatto. Control theory and fast marching techniques for brain connectivity mapping. Research Report 5845, INRIA Sophia Antipolis, 2006b.

Emmanuel Prados, Fabio Camilli, and Olivier Faugeras. A viscosity solution method for shape-from-shading without image boundary data. Mathematical Modelling and Numerical Analysis (M2AN), 40(2):393-412, 2006c.

J. Qian, Y.-T. Zhang, and H.-K. Zhao. A fast sweeping method for static convex hamilton-jacobi equations. J. Sci. Comput., 31(1-2):237-271, 2007.

L. Qingfen. Enhancement, extraction, and visualization of $3 D$ volume data. PhD thesis, Linköping University, 2003.

E. Rouy and A. Tourin. A Viscosity Solutions Approach to Shape-from-Shading. SIAM J. Numer. Anal., 29(3):867-884, June 1992.

J.A. Sethian. A fast marching level set method for monotonically advancing fronts. Proceedings of the National Academy of Sciences, 93(4):1591-1694, 1996a.

J.A. Sethian. Level Set Methods. Cambridge University Press, 1996b.

J.A. Sethian and A. Vladimirsky. Ordered upwind methods for static Hamilton-Jacobi equations: Theory and algorithms. SIAM Journal on Numerical Analysis, 41(1):325-363, 2003.

H. M. Soner. Optimal control with state space constraints. SIAM Journal Control and Optimization, 24:Part I: 552-562, Part II: 1110-1122, 1986.

D.G. Taylor and M.C. Bushell. The spatial mapping of translational diffusion coefficients by the NMR imaging technique. Phys. Med. Bio., 30:345-349, 1985.

J.D. Tournier, F. Calamante, D.G. Gadian, and A. Connelly. Diffusion-weighted magnetic resonance imaging fibre tracking using a front evolution algorithm. NeuroImage, 20:276$288,2003$.

Y.-H. Tsai, L.-T. Cheng, S. Osher, and H.-K. Zhao. Fast sweeping algorithms for a class of Hamilton-Jacobi equations. SIAM J. Numer. Anal., 41(2):673-694, 2003a. 
Y-H. Tsai, Y. Giga, and S. Osher. A level set approach for computing discontinuous solutions of hamilton-jacobi equations. Math. Comput., 72(241):159-181, 2003b.

J.N. Tsitsiklis. Efficient algorithms for globally optimal trajectories. IEEE Trans. Aut. Control, 40:1528-1538, 1995.

D. Tuch. Diffusion MRI of Complex Tissue Structure. PhD thesis, Harvard University and Massachusetts Institute of Technology, 2002.

B. Vemuri, Y. Chen, M. Rao, T. McGraw, T. Mareci, and Z. Wang. Fiber tract mapping from diffusion tensor MRI. In IEEE Workshop on Variational and Level Set Methods, page 81, July 2001.

R. Venkataraman, R. Holsapple, and D. Doman. Optimal control problems on Riemannian manifolds: Theory and applications. Technical Report A433804, Air force research lab Wright-Patterson AFB OH air vehicles directorate, 2002.

V.J. Wedeen, T.L. Davis, R.M. Weisskoff, R. Tootell, B.R. Rosen, and J.W. Belliveau. White matter connectivity explored by MRI. In Proceedings of the First International Conference for Functional Mapping of the Human Brain, page P1.36, 1995.

L. Yatziv, A. Bartesaghi, and G. Sapiro. O(N) implementation of the fast marching algorithm. Journal of computational physics, 212:393-399, 2006.

E. Yörük, B. Acar, and R. Bammer. A physical model for DT-MRI based connectivity map computation. In Medical Image Computing and Computer-Assisted Intervention, pages 213-220, 2005.

F. Zhang, C. Goodlett, E. Hancock, and G. Gerig. Probabilistic fiber tracking using particle filtering. In International Conference on Medical Image Computing and Computer-Assisted Intervention, pages 144-152, 2007.

\section{Nomenclature}

$\left[\left[D_{x}^{-1}\right]\right]^{i}$ Matrix $D_{x}^{-1}$ without the $i^{\text {th }}$ row and $i^{\text {th }}$ column. 
$\alpha \quad$ Control function $\Omega \mapsto \mathcal{A}$.

$\alpha^{\star} \quad$ Optimal control.

D Diffusion tensor ( $D_{x}$ is also used to denote the spatial variation of the tensors).

$\Delta_{\mathcal{M}} \quad$ Laplace-Beltrami operator.

$\gamma_{0} \quad$ Geodesic curve.

$\Gamma_{x, y} \quad$ Set of curves linking $x$ and $y$.

$\mathcal{A} \quad$ Set of admissible controls $a$.

$\mathcal{C}(x) \quad$ Connectivity measure at $x$.

$\mathcal{L}(x, y)$ Length of a curve $\gamma$ between $x$ and $y$.

$\mathcal{M}$ Riemannian manifold.

$\mathcal{S}_{R}(0,1)$ Riemannian unit sphere.

$\mathcal{T} \quad$ Target voxel for a given trajectory (geodesic curve).

$\mu(x) \quad$ Mean value of $\mathcal{C}(x)$ along the optimal trajectory starting at $x$,

$\nabla u \quad$ Differential of the function $u$.

$\nabla_{\mathbf{x}^{i}}^{ \pm} \quad$ Forward/backward approximation of the differential in the direction $\mathbf{x}^{i}$.

$|\cdot|_{E} \quad$ Euclidean norm.

$|\cdot|_{R} \quad$ Riemannian norm.

$\Omega \quad$ Data acquisition grid (subset of $\mathbb{R}^{3}$ ).

$\rho \quad$ Size of the grid $\left(h_{1}, h_{2}, h_{3}\right)$.

$\sigma(x) \quad$ Standard deviation of $\mathcal{C}(x)$ along the optimal trajectory starting at $x$.

$\tau_{x}^{\star} \quad$ Length of the optimal trajectory $\xi_{x}^{\star}$.

$\operatorname{div} v \quad$ Intrinsic divergence of the vector field $v$. 
$\operatorname{grad} u$ Intrinsic gradient of the function $u$.

$\tilde{p}^{i} \quad$ Vector $p \in \mathbb{R}^{n}$ without $i^{\text {th }}$ component.

$\xi \quad$ Candidate trajectory $\mathbb{R}^{+} \mapsto \Omega$ between two points.

$\xi_{x}^{\star} \quad$ Optimal trajectory from $x$.

$\xi_{x, \alpha} \quad$ Trajectory starting from $x$ and control by $\alpha$.

$\left\{e_{i}\right\} \quad$ Canonical basis of $\mathbb{R}^{n}$.

$A_{x} \quad$ Square root of $D_{x}$.

$B_{E}(0,1)$ Euclidean unit ball.

$B_{R}(0,1)$ Riemannian unit ball.

f Dynamics $\Omega \times \mathcal{A} \mapsto \mathbb{R}^{3}$ (trajectory tangent).

$f^{\star} \quad$ Optimal dynamics.

G Riemannian metric with components $g_{i j}$.

$H(x, p)$ Hamiltonian $\mathcal{M} \times T^{\star} \mathcal{M} \rightarrow \mathbb{R}$.

$H^{\star}(x, a)$ Legendre transform of the Hamiltonian $H(x, p)$.

$l \quad$ Cost function $\Omega \times \mathcal{A} \mapsto \mathbb{R}$.

$p \quad$ Short notation for the differential $\nabla u$.

$P_{s_{i}} \quad$ Approximation of the $i^{\text {th }}$ component of the differential of $U$

$s_{i} \quad$ Sign of the $i^{\text {th }}$ component of the dynamics.

$T_{x y} \quad$ Time of arrival at $y$ of the geodesic starting from $x$.

$U \quad$ Approximation of the geodesic distance function.

$V \quad$ Value function.

$x, y \quad$ Points on the grid (voxel). 


\section{List of Figures}

1 Approximation of the geodesic (a), Topological problem (b) . . . . . . . . . 35

2 Synthetic (left) and human brain (right) DTI datasets. . . . . . . . . . . . 36

3 Distance function for synthetic and human brain datasets $\ldots \ldots \ldots$

4 Geodesic paths originating from the occipital area . . . . . . . . . . . 38

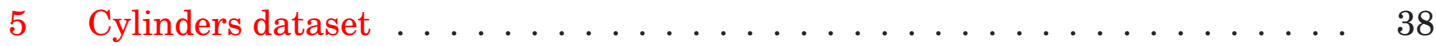

6 Topological inconsistency in the occipital cortex . . . . . . . . . . . . . . . . 40

$7 \quad$ Optimal dynamics estimation $\ldots \ldots \ldots \ldots \ldots \ldots$. . . . . . . . . . . 41

8 Synthetic dataset: Connectivity map and most likely connections . . . . . . . . 43

9 Real dataset: Connectivity map and most likely connections . . . . . . . . . . . 44 


\section{List of Tables}

$1 \quad$ Statistics on $|\operatorname{grad} u|$ for synthetic and real DTI datasets. . . . . . . . . . . . . 39 